\title{
Restoration of Poissonian Images Using Alternating Direction Optimization
}

\author{
Mário A. T. Figueiredo, Fellow, IEEE, and José M. Bioucas-Dias, Member, IEEE
}

\begin{abstract}
Much research has been devoted to the problem of restoring Poissonian images, namely for medical and astronomical applications. However, the restoration of these images using state-of-the-art regularizers (such as those based upon multiscale representations or total variation) is still an active research area, since the associated optimization problems are quite challenging. In this paper, we propose an approach to deconvolving Poissonian images, which is based upon an alternating direction optimization method. The standard regularization [or maximum a posteriori (MAP)] restoration criterion, which combines the Poisson log-likelihood with a (nonsmooth) convex regularizer (log-prior), leads to hard optimization problems: the log-likelihood is nonquadratic and nonseparable, the regularizer is nonsmooth, and there is a nonnegativity constraint. Using standard convex analysis tools, we present sufficient conditions for existence and uniqueness of solutions of these optimization problems, for several types of regularizers: total-variation, frame-based analysis, and frame-based synthesis. We attack these problems with an instance of the alternating direction method of multipliers (ADMM), which belongs to the family of augmented Lagrangian algorithms. We study sufficient conditions for convergence and show that these are satisfied, either under total-variation or frame-based (analysis and synthesis) regularization. The resulting algorithms are shown to outperform alternative state-of-the-art methods, both in terms of speed and restoration accuracy.
\end{abstract}

Index Terms-Alternating direction methods, augmented Lagrangian, convex optimization, image deconvolution, image restoration, Poisson images.

\section{INTRODUCTION}

A LARGE fraction of the work on image denoising, restoration, and reconstruction has been devoted to developing regularizers (priors, from a Bayesian point of view) to deal with the presence of noise and/or the ill-conditioned or ill-posed nature of the observation operator, and to devising efficient algorithms to solve the resulting optimization problems. Much of that work assumes linearity of the observation operator (e.g., the convolution with some point spread function, the acquisition of tomographic projections, or simply an identity in the

Manuscript received January 13, 2010; revised April 05, 2010; accepted June 02, 2010. Date of publication June 28, 2010; date of current version November 17, 2010. This work was supported in part by the Fundação para a Ciencia e Tecnologia (FCT), Portuguese Ministry of Science and Higher Education, under Projects POSC/EEA-CPS/61271/2004, PTDC/EEA-TEL/104515/2008, and UTAustin/MAT/0009/2008. The associate editor coordinating the review of this manuscript and approving it for publication was Dr. Yongyi Yang.

The authors are with the Instituto de Telecomunicações, Instituto Superior Técnico, 1049-001 Lisboa, Portugal. (e-mail: mario.figueiredo@1x.it.pt; bioucas@1x.it.pt).

Color versions of one or more of the figures in this paper are available online at http://ieeexplore.ieee.org.

Digital Object Identifier 10.1109/TIP.2010.2053941 case of denoising) and the presence of additive Gaussian noise. For this classical scenario, recent state-of-the-art methods adopt nonsmooth convex regularizers, such as total-variation or the $\ell_{1}$ norm of frame coefficients; the resulting optimization problems are convex but nonsmooth, of very high dimensionality, and have stimulated a considerable amount of research on special purpose algorithms (see [4], [5], [16], [26], [53], [59], and the many references therein).

The algorithms developed for linear operators and Gaussian noise cannot be directly applied to other observation models, such as the Poisson case considered in this paper. Poissonian image models are well studied and highly relevant in fields such as astronomical [51], biomedical [18], [24], [42], [46], [55], [57], and photographic imaging [27].

Comprehensive reviews of Poissonian image restoration methods, including the classical Richardson-Lucy (RL) algorithm, can be found in [46] and [51]. Regularized variants of the RL algorithm have been proposed in [18] (with total-variation) and in [52] (with wavelet-based regularization). Other wavelet-based approaches to Poissonian inverse problems include [2] and [43] (both based upon the wavelet-vaguelette decomposition [19]), [8] (based upon the vaguelette-wavelet decomposition [1]), and [41] (based upon a cross-validation filter design [40]). A frame-based state-of-the-art method recently proposed in [20] uses the Anscombe variance stabilizing transform, which allows replacing a linear observation model with Poisson (nonadditive) noise by a nonlinear observation model with additive noise. Other multiscale approaches to handling imaging inverse problems with Poissonian observations can be found in [42], [55], [57], and [58]. A method based upon local polynomial approximations was introduced in [27]. Finally, there is also a vast literature on the use of expectation-maximization (EM) methods for image reconstruction from Poisson data using penalized maximum likelihood (PML) criteria; a representative publication (where many earlier references can be found) is [24].

The standard criterion for deconvolution of Poissonian images consists of a convex constrained optimization problem: the objective function includes the so-called data term, which is convex and smooth, but not quadratic, plus a convex nonsmooth regularizer (the log-likelihood and log-prior, from a Bayesian inference perspective), and a constraint forcing the solution to be nonnegative. Although the problem is convex, its very high dimensionality (when dealing with images) usually rules out the direct application of off-the-shelf optimization algorithms.

Furthermore, the Poisson log-likelihood, which is nonquadratic and nonseparable (except in the pure denoising case) raises several difficulties to the current state-of-the-art image deconvolution algorithms. More specifically, the Poisson 
log-likelihood does not have a Lipschitz-continuous gradient, a sufficient condition for the applicability (with guaranteed convergence) of algorithms of the forward-backward splitting (FBS) family [16], [20], [59]. If, nevertheless, an FBS method is applied, it is well known to be slow, specially if the observation operator is severely ill-conditioned, a fact which has stimulated recent research on faster methods [4], [5], [59]; these faster algorithms also require the log-likelihood to have a Lipschitz-continuous gradient, which is not the case with Poissonian observations.

In this paper, we propose a new approach to tackle the optimization problem referred to in the previous paragraph. The proposed algorithm is based upon an instance of the alternating direction method of multipliers (ADMM) [21], [28], [29], which belongs to the family of augmented Lagrangian methods [39]. For this reason, we call it PIDAL (Poisson image deconvolution by augmented Lagrangian). Although the proposed approach is related to the recent split-Bregman (SB) technique [30], our splitting strategy and resulting algorithm are quite different from the one in [30] (which, moreover, is not adequate for Poissonian image models). This paper is an extension of our much shorter and preliminary work [25]; another extension of that earlier work of ours has recently appeared in [50]. Finally, we should mention that related work on a different nonadditive noise model (multiplicative Gamma-distributed noise) can be found in [3], [6], [54].

In recent work, Douglas-Rachford splitting (DRS) methods were proposed to attack problems in which log-likelihood the does not have a Lipschitz-continuous gradient [15], [54]. In fact, the ADMM is closely related to DRS methods [21], [23], so the method proposed in this paper can also be interpreted from a DRS viewpoint.

In this paper, we will consider three types of regularization: total variation [9], [44] and both frame-based analysis and frame-based synthesis formulations [22]. In Section II, after presenting these three formulations, we derive sufficient conditions for existence and uniqueness of solutions of the corresponding optimization problems. The ADMM framework is reviewed in Section III, where we also introduce the particular variant that is suitable for a linear combination of several convex functions, which is the form of the objective function in hand. In Sections IV and V, we instantiate the proposed variant of ADMM to the three types of regularizers considered and provide sufficient conditions for convergence. Finally, the effectiveness of the resulting algorithm is illustrated in comparison with current state-of-the-art alternatives [18], [20], [27], [50], [52], via a set of experiments reported in Section VI.

\section{PROBLEM FORMULATION}

In this section, we begin by reviewing the derivation of the standard log-likelihood resulting from assuming that the observations are Poisson distributed with a mean intensity linearly related with the underlying image to be estimated. Then, we present three different regularization/Bayesian criteria, using synthesis and analysis formulations [22], and study existence/uniqueness of the corresponding solutions.

\section{A. Linear/Poisson Observation Model}

Let $\mathbf{y}=\left(y_{1}, \ldots, y_{m}\right) \in \mathbb{N}_{0}^{m}$ denote an $m$-vector of observed counts $\left(\mathbb{N}_{0}=\mathbb{N} \cup\{0\}\right)$, assumed to be a sample of a random vector $\mathbf{Y}=\left(Y_{1}, \ldots, Y_{m}\right)$ of $m$ independent Poisson variables, with probability distribution

$$
P[\mathbf{Y}=\mathbf{y} \mid \boldsymbol{\lambda}]=\prod_{i=1}^{m} \frac{\lambda_{i}^{y_{i}} e^{-\lambda_{i}}}{y_{i} !}
$$

where $\boldsymbol{\lambda}=\left(\lambda_{1}, \ldots, \lambda_{m}\right) \in \mathbb{R}_{+}^{m}\left(\mathbb{R}_{+}\right.$denotes the nonnegative reals) is the underlying mean (intensity) vector, assumed to be a linear observation of an unknown image $\mathbf{x}$, i.e.,

$$
\lambda=\mathbf{K x}
$$

where $\mathbf{K}$ the observation operator, which in our finite dimensional setting is simply a matrix $\mathbf{K} \in \mathbb{R}^{m \times n}$. This matrix may model a convolution or some other linear observation mechanism, such as emission tomography. So that the underlying unknown $\mathbf{x}$ can also have the meaning of intensity, it is commonly assumed that $\mathbf{x} \in \mathbb{R}_{+}^{n}$. It is usually further assumed that all the elements of $\mathbf{K}$ are nonnegative [18], [24], [51]. When dealing with images, we adopt the usual vector notation obtained by stacking the pixels into a vector, in lexicographic order.

Combining (1) and (2) and taking logarithms leads to the negative log-likelihood function [18], [51]

$$
\begin{aligned}
-\log P[\mathbf{Y}=\mathbf{y} \mid \mathbf{x}]= & \sum_{i=1}^{m}(\mathbf{K} \mathbf{x})_{i}-y_{i} \log \left((\mathbf{K} \mathbf{x})_{i}\right) \\
& +\log \left(y_{i} !\right) \\
= & \mathcal{L}(\mathbf{K} \mathbf{x}) \\
= & (\mathcal{L} \circ \mathbf{K})(\mathbf{x})
\end{aligned}
$$

where $(\mathbf{v})_{i}$ (or $v_{i}$ ) denotes the $i$ th component of some vector $\mathbf{v}$ and $\mathcal{L}: \mathbb{R}^{m} \rightarrow \overline{\mathbb{R}}=\mathbb{R} \cup\{-\infty,+\infty\}$ is the negative loglikelihood function for the case $\mathbf{K}=\mathbf{I}$, that is

$$
\mathcal{L}(\mathbf{z})=\sum_{i=1}^{m}-y_{i} \log \left(z_{i}\right)+z_{i}+\log \left(y_{i} !\right)
$$

Dealing with the particular case $z_{i}=0$ requires some care, because of the presence of the logarithm. Seen as function of $\mathbf{z}$ to be used in a minimization problem, it is convenient to write the negative log-likelihood function as

$$
\mathcal{L}(\mathbf{z})=C+\sum_{i=1}^{m} \xi\left(z_{i}, y_{i}\right)
$$

where $C=\sum_{i} \log \left(y_{i} !\right)$ is a finite (recall that $0 !=1$ ) irrelevant (independent of $\mathbf{z}$ ) constant and $\xi: \mathbb{R} \times \mathbb{N}_{0} \rightarrow \overline{\mathbb{R}}$ is defined as

$$
\xi(z, y)=z+\iota_{\mathbb{R}_{+}}(z)-y \log \left(z_{+}\right)
$$

where $\iota_{S}$ is the indicator function of set $S$

$$
\begin{gathered}
\iota_{S}(z)= \begin{cases}0, & \Leftarrow z \in S \\
+\infty, & \Leftarrow z \notin S\end{cases} \\
z_{+}=\max \{0, z\}, \log (0)=-\infty, \text { and } 0 \log (0)=0 .
\end{gathered}
$$


The following two propositions characterize $\xi$ as a function of its first argument, as well as $\mathcal{L}$ and $\mathcal{L} \circ \mathbf{K}$, in terms of the key concepts of convex analysis (see Appendix A).

Proposition 1: For any $y \in \mathbb{N}_{0}$ the function $\xi(\cdot, y): \mathbb{R} \rightarrow \overline{\mathbb{R}}$ is proper, lower semicontinuous (lsc), coercive, and convex. If $y>0$, then $\xi(\cdot, y)$ is also strictly convex.

Proof: For $y=0, \xi(z, 0)=z+\iota_{\mathbb{R}_{+}}(z)$, thus, $\xi(\cdot, 0)$ is the sum of the identity function with $\iota_{\mathbb{R}_{+}}$, which are both proper, lsc, coercive, and convex. For any $y>0, \xi(z, y)=$ $z+\iota_{\mathbb{R}_{+}}(z)-y \log \left(z_{+}\right)$; since $y \log \left((\cdot)_{+}\right)$is also proper, lsc, coercive, and convex, so is $\xi(\cdot, y)$. Finally, if $y>0, y \log \left((\cdot)_{+}\right)$ is strictly convex (see the definition in Appendix A), thus, $\xi(\cdot, y)$ is also strictly convex.

Proposition 2: Function $\mathcal{L}$ is proper, lsc, coercive, and convex. If $y_{i} \neq 0$, for $i=1, \ldots, m, \mathcal{L}$ is also strictly convex. Function $\mathcal{L} \circ \mathbf{K}$ is proper, 1sc, and convex. Function $\mathcal{L} \circ \mathbf{K}$ is coercive if $\mathbf{K}$ is injective. Function $\mathcal{L} \circ \mathbf{K}$ is strictly convex if $\mathbf{K}$ is injective and $y_{i} \neq 0$, for $i=1, \ldots, m$.

Proof: Function $\mathcal{L}$ is the sum of proper, lsc, coercive, convex functions. If $y_{i} \neq 0$, for $i=1, \ldots, m$, the functions in the sum are also strictly convex, thus, $\mathcal{L}$ is also strictly convex. Function $\mathcal{L} \circ \mathbf{K}$ is the composition of a proper, lsc, convex functions with a linear function, thus, it is proper, lsc, and convex. If $\mathbf{K}$ is injective, its null set is the zero vector, thus, $\lim _{\|\mathbf{x}\| \rightarrow+\infty}\|\mathbf{K x}\|=+\infty$, thus, $\mathcal{L} \circ \mathbf{K}$ is coercive. Finally, if $\mathbf{K}$ is injective and $y_{i} \neq 0$, for $i=1, \ldots, m, \mathcal{L}$ is strictly convex, thus, so is $\mathcal{L} \circ \mathbf{K}$.

\section{B. Regularization Criteria: Total Variation and Frame-Based Analysis and Synthesis Formulations}

Under a maximum a posteriori (MAP) or regularization criterion, the image estimate is obtained by solving a variational problem: minimizing an objective function, which includes the log-likelihood term plus a regularizer [25], [34], [51], under a positivity constraint. We will now describe three possible ways of building such an objective function.

1) Total Variation Regularization: A standard choice for regularization of digital image restoration/reconstruction problems is the isotropic discrete TV regularizer [9]

$$
\operatorname{TV}(\mathbf{x})=\sum_{s=1}^{n} \sqrt{\left(\Delta_{s}^{h} \mathbf{x}\right)^{2}+\left(\Delta_{s}^{v} \mathbf{x}\right)^{2}}
$$

where $\left(\Delta_{s}^{h} \mathbf{x}\right.$ and $\left.\Delta_{s}^{v} \mathbf{x}\right)$ denote the horizontal and vertical firstorder differences at pixel $s$, respectively. This regularizer is a discrete version of the TV regularizer proposed in [44]. The resulting optimization problem is

$$
\min _{\mathbf{x}} L^{\mathrm{TV}}(\mathbf{x})
$$

with

$$
L^{\mathrm{TV}}(\mathbf{x})=\mathcal{L}(\mathbf{K} \mathbf{x})+\tau \mathrm{TV}(\mathbf{x})+\iota_{\mathbb{R}_{+}^{n}}(\mathbf{x})
$$

where $\tau \in \mathbb{R}_{+}$is the regularization parameter and the role of $\iota \mathbb{R}_{+}^{n}$, the indicator of the first orthant, is to impose the nonnegativity constraint on the estimate. The next proposition concerns the existence and uniqueness of minimizers of $L^{\mathrm{TV}}$.

Proposition 3: Consider the function $L^{\mathrm{TV}}$ defined in (10). a) $L^{\mathrm{TV}}$ is proper, lsc, and convex.

b) If the intersection of the space of constant images $\{x=$ $\alpha(1,1, \ldots, 1), \alpha \in \mathbb{R}\}$ (which is the null space of TV) with the null space of $\mathbf{K}$ is just the zero vector, then $L^{\mathrm{TV}}$ is coercive, and (9) has a solution.

c) If $\mathbf{K}$ is injective, then (9) has a solution.

d) If $\mathbf{K} \in \mathbb{R}_{+}^{m \times n}$, and at least one element of $\mathbf{K}$ is strictly positive, then (9) has a solution.

e) If $\mathbf{K}$ is injective and $y_{i} \neq 0$, for $i=1, \ldots, m$, then $L^{\mathrm{TV}}$ is coercive and strictly convex, thus, there is a unique solution.

Proof:

a) the functions $\iota_{\mathbb{R}_{+}^{n}}$, TV, and $\mathcal{L} \circ \mathbf{K}$ (Proposition 2) are proper, 1sc, and convex, thus, so is their sum;

b) similar to [10];

c) if $\mathbf{K}$ is injective, its null space is just the zero vector, thus, $\mathcal{L} \circ \mathbf{K}$ and $L^{\mathrm{TV}}$ are coercive;

d) if all the elements of $\mathbf{K}$ are nonnegative and at least one is positive, then the constant vector $(1,1, \ldots, 1)$ doesn't belong to the null space of $\mathbf{K}$ and the result follows from b);

e) if $\mathbf{K}$ is injective and $y_{i} \neq 0$, for $i=1, \ldots, m, \mathcal{L} \circ \mathbf{K}$ is strictly convex (Proposition 2), thus, so is $L^{\mathrm{TV}}$ and its minimizer is unique.

2) Frame Analysis Regularization: The use of a regularizer which is a direct function of the unknown image [as in (9)-(10)] corresponds to a so-called analysis-based prior/regularizer [22]. Another well-known type of analysis-based regularization penalizes the norm (typically $\ell_{1}$ ) of the representation coefficients of $\mathbf{x}$ on some wavelet basis or tight frame (e.g., of wavelets, curvelets, or other multiscale system), given by $\mathbf{P x}$, where $\mathbf{P}$ is the analysis operator associated with the frame [36]. This approach leads to the following optimization problem:

$$
\min _{\mathbf{x}} L^{\mathrm{FA}}(\mathbf{x})
$$

where FA stands for frame analysis and

$$
L^{\mathrm{FA}}(\mathbf{x})=\mathcal{L}(\mathbf{K} \mathbf{x})+\tau\|\mathbf{P} \mathbf{x}\|_{1}+\iota_{\mathbb{R}_{+}^{n}}(\mathbf{x})
$$

as shown previously, $\tau$ is the regularization parameter and $\iota_{\mathbb{R}_{+}^{n}}$ imposes the nonnegativity constraint on the estimate. The next proposition addresses the existence and uniqueness of minimizers of $L^{\mathrm{FA}}$.

Proposition 4: Consider the function $L^{\mathrm{FA}}$ defined in (12).

a) $L^{\mathrm{FA}}$ is proper, lsc, convex, and coercive, thus, has a minimizer.

b) If $\mathbf{K}$ is injective and $y_{i} \neq 0$, for $i=1, \ldots, m$, then $L^{\mathrm{FA}}$ is strictly convex with a unique minimizer.

Proof:

a) The functions $\iota_{\mathbb{R}_{+}^{n}},\|\cdot\|_{1} \circ \mathbf{P}$, and $\mathcal{L} \circ \mathbf{K}$ (Proposition 2) are proper, lsc, and convex, thus, so is their sum. Furthermore, since $\mathbf{P}$ is the analysis operator of a tight frame, its null space is simply the zero vector, thus $\|\cdot\|_{1} \circ \mathbf{P}$ is coercive.

b) If $\mathbf{K}$ is injective and $y_{i} \neq 0$, for $i=1, \ldots, m, \mathcal{L} \circ \mathbf{K}$ is strictly convex (Proposition 2), thus, so is $L^{\mathrm{FA}}$ and its minimizer is unique. 


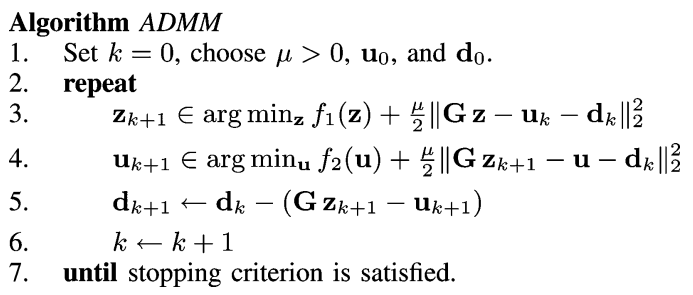

Fig. 1. ADMM algorithm.

3) Frame Synthesis Regularization: Finally, another wellknown class of approaches is known as synthesis-based [22]. Here, the unknown image is represented on a frame and then the coefficients of this representation are estimated from the observed data, under some regularizer. With $\mathbf{W} \in \mathbb{R}^{n \times d}$ denoting the synthesis matrix of the frame, the image is written as $\mathbf{x}=\mathbf{W} \mathbf{s}$, where $\mathbf{s}$ is the vector of representation coefficients, and the resulting optimization problem is

$$
\min _{\mathbf{s}} L^{\mathrm{FS}}(\mathbf{s})
$$

where FS stands for frame synthesis and

$$
L^{\mathrm{FS}}(\mathbf{s})=\mathcal{L}(\mathbf{K W} \mathbf{s})+\tau\|\mathbf{s}\|_{1}+\iota_{\mathbb{R}_{+}^{n}}(\mathbf{W} \mathbf{s}) .
$$

Naturally, the indicator function $\iota_{\mathbb{R}_{+}^{n}}$ forcing the image estimate to be nonnegative is applied to the image $\mathbf{W s}$ and not its coefficients. The next proposition addresses the existence and uniqueness of minimizers of $L^{\mathrm{FS}}$.

Proposition 5: Consider the function $L^{\mathrm{FS}}$ defined in (14).

a) $L^{\mathrm{FS}}$ is proper, lsc, convex, and coercive, thus, has a minimizer.

b) If $\mathbf{K W}$ is injective and $y_{i} \neq 0$, for $i=1, \ldots, m$, then $L^{\mathrm{FS}}$ is strictly convex with a unique minimizer.

Proof:

a) The functions $\iota_{\mathbb{R}_{+}^{n}} \circ \mathbf{W},\|\cdot\|_{1}$, and $\mathcal{L} \circ \mathbf{K} \mathbf{W}$ (Proposition 2) are proper, lsc, and convex, thus, so is their sum. Furthermore, since $\|\cdot\|_{1}$ is coercive, $L^{\mathrm{FS}}$ is coercive.

b) Same proof as that of Proposition 4 (b).

\section{Alternating Direction Method OF MULTIPLIERS (ADMM)}

\section{A. Standard ADMM}

The key tool in this paper is the alternating direction method of multipliers (ADMM) [21], [28], [29]. Consider an unconstrained problem of the form

$$
\min _{\mathbf{z} \in \mathbb{R}^{d}} f_{1}(\mathbf{z})+f_{2}(\mathbf{G z})
$$

where $f_{1}: \mathbb{R}^{d} \rightarrow \overline{\mathbb{R}}, f_{2}: \mathbb{R}^{p} \rightarrow \overline{\mathbb{R}}$, and $\mathbf{G} \in \mathbb{R}^{p \times d}$. The ADMM for this problem is defined in Fig. 1.

For later reference, we now recall a theorem by Eckstein and Bertsekas in which convergence of (a generalized version of) ADMM is shown.

Theorem 1 (Eckstein-Bertsekas, [21]): Consider problem (15), where $\mathbf{G} \in \mathbb{R}^{p \times d}$ has full column rank and $f_{1}: \mathbb{R}^{d} \rightarrow \overline{\mathbb{R}}$ and $f_{2}: \mathbb{R}^{p} \rightarrow \overline{\mathbb{R}}$ are closed, proper, convex functions. Consider arbitrary $\mu>0$ and $\mathbf{u}_{0}, \mathbf{d}_{0} \in \mathbb{R}^{p}$. Let $\left\{\eta_{k} \geq 0, k=0,1, \ldots\right\}$ and $\left\{\rho_{k} \geq 0, k=0,1, \ldots\right\}$ be two sequences such that

$$
\sum_{k=0}^{\infty} \eta_{k}<\infty \text { and } \sum_{k=0}^{\infty} \rho_{k}<\infty .
$$

Consider three sequences $\left\{\mathbf{z}_{k} \in \mathbb{R}^{d}, k=0,1, \ldots\right\},\left\{\mathbf{u}_{k} \in\right.$ $\left.\mathbb{R}^{p}, k=0,1, \ldots\right\}$, and $\left\{\mathbf{d}_{k} \in \mathbb{R}^{p}, k=0,1, \ldots\right\}$ that satisfy

$$
\begin{aligned}
\left\|\mathbf{z}_{k+1}-\arg \min _{\mathbf{z}} f_{1}(\mathbf{z})+\frac{\mu}{2}\right\| \mathbf{G z}-\mathbf{u}_{k}-\mathbf{d}_{k}\left\|_{2}^{2}\right\| & \leq \eta_{k} \\
\left\|\mathbf{u}_{k+1}-\arg \min _{\mathbf{u}} f_{2}(\mathbf{u})+\frac{\mu}{2}\right\| \mathbf{G z}_{k+1}-\mathbf{u}-\mathbf{d}_{k}\left\|_{2}^{2}\right\| & \leq \rho_{k}
\end{aligned}
$$

and

$$
\mathbf{d}_{k+1}=\mathbf{d}_{k}-\left(\mathbf{G z}_{k+1}-\mathbf{u}_{k+1}\right) .
$$

Then, if (15) has a solution, say $\mathbf{z}^{*}$, the sequence $\left\{\mathbf{z}_{k}\right\}$ converges to $\mathbf{z}^{*}$. If (15) does not have a solution, then at least one of the sequences $\left\{\mathbf{u}_{k}\right\}$ or $\left\{\mathbf{d}_{k}\right\}$ diverges.

According to Theorem 1, it is not necessary to exactly solve the minimizations in lines 3 and 4 of ADMM: as long as the sequences of errors are absolutely summable, convergence is not compromised. As shown in Section IV-D, this fact is quite relevant in designing instances of ADMM, when these minimizations lack closed form solutions.

The proof of Theorem 1 is based upon the equivalence between ADMM and the DRS method applied to the dual of problem (15). For recent and comprehensive reviews of ADMM, DRS, and their relationship with Bregman and split-Bregman methods, see [23] and [49].

\section{B. Variant of $A D M M$}

Notice that the ADMM and the associated convergence theorem presented in the previous subsection apply to objective functions of the form (15), i.e., which are the sum of two functions. The fact that our objective functions (9), (11), and (13) involve more than two terms requires finding a way of mapping an objective with more than two terms into (15) so that the resulting ADMM is easily applicable and the conditions of Theorem 1 still hold.

Consider a generalization of problem (15), where instead of two functions, we have $J$ functions, that is

$$
\min _{\mathbf{z} \in \mathbb{R}^{d}} \sum_{j=1}^{J} g_{j}\left(\mathbf{H}^{(j)} \mathbf{z}\right)
$$

where $g_{j}: \mathbb{R}^{p_{j}} \rightarrow \overline{\mathbb{R}}$ are closed, proper, convex functions, and $\mathbf{H}^{(j)} \in \mathbb{R}^{p_{j} \times d}$ are arbitrary matrices. The minimization problem (17) can be written as (15) using the following correspondences:

$$
\begin{gathered}
f_{1}=0 \\
\mathbf{G}=\left[\begin{array}{c}
\mathbf{H}^{(1)} \\
\vdots \\
\mathbf{H}^{(J)}
\end{array}\right] \in \mathbb{R}^{p \times d}
\end{gathered}
$$


where $p=p_{1}+\cdots+p_{J}$, and $f_{2}: \mathbb{R}^{p \times d} \rightarrow \overline{\mathbb{R}}$ given by

$$
f_{2}(\mathbf{u})=\sum_{j=1}^{J} g_{j}\left(\mathbf{u}^{(j)}\right)
$$

where $\mathbf{u}^{(j)} \in \mathbb{R}^{p_{j}}$ and $\mathbf{u}=\left[\left(\mathbf{u}^{(1)}\right)^{T}, \ldots,\left(\mathbf{u}^{(J)}\right)^{T}\right]^{T} \in \mathbb{R}^{p}$.

We are now in position to apply ADMM. The resulting algorithm has exactly the same structure as the one in Fig. 1 with

$$
\mathbf{d}_{k}=\left[\begin{array}{c}
\mathbf{d}_{k}^{(1)} \\
\vdots \\
\mathbf{d}_{k}^{(J)}
\end{array}\right], \quad \mathbf{u}_{k}=\left[\begin{array}{c}
\mathbf{u}_{k}^{(1)} \\
\vdots \\
\mathbf{u}_{k}^{(J)}
\end{array}\right]
$$

The fact that $f_{1}=0$ turns Step 3 of the algorithm into a simple quadratic minimization problem, which has a unique solution if $\mathbf{G}$ has full column rank

$$
\begin{aligned}
\arg \min _{\mathbf{z}}\left\|\mathbf{G} \mathbf{z}-\boldsymbol{\zeta}_{k}\right\|_{2}^{2} & =\left(\mathbf{G}^{T} \mathbf{G}\right)^{-1} \mathbf{G}^{T} \boldsymbol{\zeta}_{k} \\
& =\left[\sum_{j=1}^{J}\left(\mathbf{H}^{(j)}\right)^{T} \mathbf{H}^{(j)}\right]-1 \sum_{j=1}^{J}\left(\mathbf{H}^{(j)}\right)^{T} \boldsymbol{\zeta}_{k}^{(j)}
\end{aligned}
$$

where $\boldsymbol{\zeta}_{k}=\mathbf{u}_{k}+\mathbf{d}_{k}$ (and, naturally, $\boldsymbol{\zeta}_{k}^{(j)}=\mathbf{u}_{k}^{(j)}+\mathbf{d}_{k}^{(j)}$ ) and the second equality results from the particular structure of $\mathbf{G}$ in (18).

Furthermore, our particular way of mapping problem (17) into problem (15) allows decoupling the minimization in Step 4 of ADMM into a set of $J$ independent ones. In fact

$$
\mathbf{u}_{k+1} \leftarrow \arg \min _{\mathbf{u}} f_{2}(\mathbf{u})+\frac{\mu}{2}\left\|\mathbf{G} \mathbf{z}_{k+1}-\mathbf{u}-\mathbf{d}_{k}\right\|_{2}^{2}
$$

which can be written as

$$
\begin{aligned}
& {\left[\begin{array}{c}
\mathbf{u}_{k+1}^{(1)} \\
\vdots \\
\mathbf{u}_{k+1}^{(J)}
\end{array}\right] \leftarrow \arg \min _{\mathbf{u}^{(1)}, \ldots, \mathbf{u}^{(J)}} g_{1}\left(\mathbf{u}^{(1)}\right)+\cdots+g_{J}\left(\mathbf{u}^{(J)}\right)} \\
& +\frac{\mu}{2}\left\|\left[\begin{array}{c}
\mathbf{H}^{(1)} \\
\vdots \\
\mathbf{H}^{(J)}
\end{array}\right] \mathbf{z}_{k+1}-\left[\begin{array}{c}
\mathbf{u}^{(1)} \\
\vdots \\
\mathbf{u}^{(J)}
\end{array}\right]-\left[\begin{array}{c}
\mathbf{d}_{k}^{(1)} \\
\vdots \\
\mathbf{d}_{k}^{(J)}
\end{array}\right]\right\|_{2}^{2}
\end{aligned}
$$

Clearly, the minimizations with respect to $\mathbf{u}^{(1)}, \ldots, \mathbf{u}^{(J)}$ are decoupled, thus, can be solved separately, leading to

$$
\mathbf{u}_{k+1}^{(j)} \leftarrow \arg \min _{\mathbf{v} \in \mathbb{R}^{p_{j}}} g_{j}(\mathbf{v})+\frac{\mu}{2}\left\|\mathbf{v}-\mathbf{s}_{k}^{(j)}\right\|_{2}^{2}
$$

for $j=1, \ldots, J$, where $\mathbf{s}_{k}^{(j)}=\mathbf{H}^{(j)} \mathbf{z}_{k+1}-\mathbf{d}_{k}^{(j)}$.

The minimization problem in the right hand side of (22) defines the so-called Moreau proximity operator (see Appendix B for definitions and references) of $g_{j} / \mu$ (denoted as $\Psi_{g_{j} / \mu}$ ), applied to $\mathbf{s}_{k}^{(j)}$, thus

$$
\mathbf{u}_{k+1}^{(j)} \leftarrow \Psi_{g_{j} / \mu}\left(\mathbf{s}_{k}^{(j)}\right) .
$$

For some functions, the corresponding Moreau proximity operators can be computed exactly in closed form [12], [14]. A notable case is $g_{j}(\mathbf{x})=\|\mathbf{x}\|_{1}$, for which the corresponding proximity operator $\Psi_{g_{j} / \mu}$ is simply a soft threshold

$$
\begin{aligned}
\Psi_{g_{j} / \mu}(\mathbf{v}) & =\operatorname{soft}\left(\mathbf{v}, \frac{1}{\mu}\right) \\
& =\operatorname{sign}(\mathbf{v}) \odot \max \left\{|\mathbf{v}|-\left(\frac{1}{\mu}\right), 0\right\}
\end{aligned}
$$

where $\operatorname{sign}(\cdot)$ denotes the component-wise application of the sign function, $\odot$ denotes the component-wise product, $|\mathbf{v}|$ denotes the vector of absolute values of the elements of $\mathbf{v}$, and the maximum is computed in a component-wise fashion. For other functions, such as the TV regularizer, the corresponding Moreau proximity operator does not have a simple close form and needs to be computed numerically.

Some comments on the algorithm are relevant. Firstly, being exactly an ADMM, and since all the functions $g_{j}$, for $j=$ $1, \ldots, J$, are closed, proper, and convex, convergence is guaranteed if $\mathbf{G}$ has full column rank. This full column rank condition, which is also required for the inverse in (20) to exist, will be studied in the next section for each of the specific problems considered in this paper.

\section{Poissonian Image ReConstruction With TV-BASED REGULARIZATION}

\section{A. Applying ADMM}

In this section, we apply the algorithmic framework presented in Section III-B to the total-variation-based criterion (9) and (10). The objective function in (10) has the form (17) with $J=3$

$$
g_{1} \equiv \mathcal{L}, \quad g_{2} \equiv \tau \mathrm{TV}, \quad g_{3} \equiv \iota_{\mathbb{R}_{+}^{n}}
$$

and

$$
\mathbf{H}^{(1)} \equiv \mathbf{K}, \quad \mathbf{H}^{(2)} \equiv \mathbf{I}, \quad \mathbf{H}^{(3)} \equiv \mathbf{I} .
$$

The resulting ADMM algorithm, which we call PIDAL-TV (Poisson image deconvolution by augmented Lagrangian - total variation), is shown in Fig. 2.

\section{B. Implementation Aspects and Computational Cost of PIDAL-TV}

Notice that line 7 of PIDAL-TV corresponds to (20) for the particular form of matrix $\mathbf{G}$ in this problem: $\mathbf{G}=\left[\mathbf{K}^{T} \mathbf{I} \mathbf{I}\right]^{T}$ [see (25) and (26)], which is of course of full column rank. Moreover, if $\mathbf{K}$ models a periodic convolution, it is a block circulant matrix and the inversion in line 7 of the algorithm can be implemented in $O(n \log n)$ operations, via the FFT algorithm. Although this is a well-known fact, we include the derivation in the next paragraph, for the sake of completeness.

Assuming that the convolution is periodic, $\mathbf{K}$ is block-circulant with circulant blocks and can be factorized as

$$
\mathbf{K}=\mathbf{U}^{H} \mathbf{D U}
$$




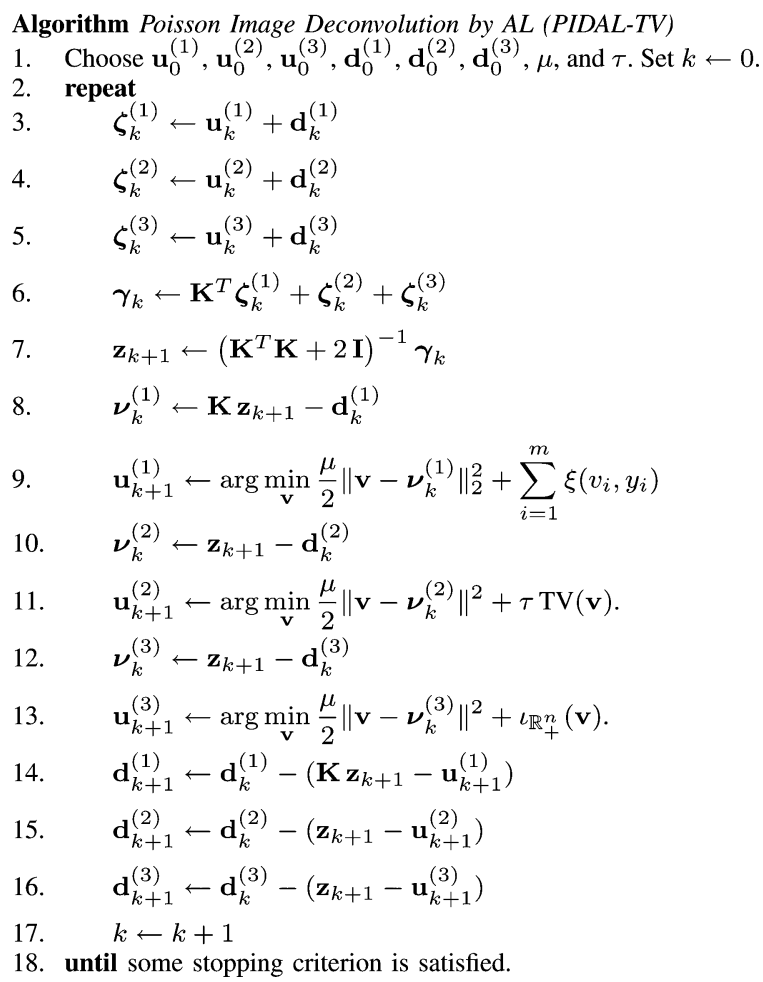

Fig. 2. PIDAL-TV algorithm.

where $\mathbf{U}$ is the matrix that represents the 2-D discrete Fourier transform (DFT), $\mathbf{U}^{H}=\mathbf{U}^{-1}$ is its inverse ( $\mathbf{U}$ is unitary, i.e., $\mathbf{U U}^{H}=\mathbf{U}^{H} \mathbf{U}=\mathbf{I}$ ), and $\mathbf{D}$ is a diagonal matrix containing the DFT coefficients of the convolution operator represented by $\mathbf{K}$. Thus, (with $\mathbf{K}^{T}=\mathbf{K}^{H}$, since $\mathbf{K}$ is a real matrix)

$$
\begin{aligned}
\left(\mathbf{K}^{T} \mathbf{K}+2 \mathbf{I}\right)^{-1} & =\left(\mathbf{U}^{H} \mathbf{D}^{*} \mathbf{D} \mathbf{U}+2 \mathbf{U}^{H} \mathbf{U}\right)^{-1} \\
& =\mathbf{U}^{H}\left(|\mathbf{D}|^{2}+2 \mathbf{I}\right)^{-1} \mathbf{U}
\end{aligned}
$$

where $(\cdot)^{*}$ denotes complex conjugate and $|\mathbf{D}|^{2}$ the squared absolute values of the entries of $\mathbf{D}$. Since $|\mathbf{D}|^{2}+2 \mathbf{I}$ is diagonal, its inversion has $O(n)$ cost. Products by $\mathbf{U}$ and $\mathbf{U}^{H}$ can be carried out with $O(n \log n)$ cost using the FFT algorithm.

The minimization in line 9 of PIDAL-TV is separable. With respect to each component, it has the form

$$
\min _{v}\left\{v+\iota_{\mathbb{R}_{+}}(v)-y \log \left(v_{+}\right)+\frac{\mu}{2}(v-\nu)^{2}\right\} .
$$

It is simple to show that the solution of (30) leads to

$$
u_{i, k+1}^{(1)}=\frac{1}{2}\left(\nu_{i, k}^{(1)}-\frac{1}{\mu}+\sqrt{\left(\nu_{i, k}^{(1)}-\frac{1}{\mu}\right)^{2}+\frac{4 y_{i}}{\mu}}\right)
$$

where $\nu_{i, k}^{(1)}$ denotes the $i$ th component of $\nu_{k}^{(1)}$. Notice that $u_{i, k+1}^{(1)}$ is always a nonnegative quantity.

The minimization in line 11 of PIDAL-TV is, by definition, the Moreau proximity operator $\Psi_{(\tau / \mu) \mathrm{TV}}: \mathbb{R}^{n} \rightarrow \mathbb{R}^{n}[16]$, which corresponds to applying TV-denoising to $\boldsymbol{\nu}_{k}^{(2)}$. In the following, we address in detail the issue of how to implement this operator and its implications to the convergence of PIDAL-TV. Suffice it to say here that most TV-denoising algorithms have $O(n)$ cost.
The minimization in line 13 of PIDAL-TV corresponds to the projection of $\boldsymbol{\nu}_{k}^{(3)}$ onto the first orthant, thus

$$
\mathbf{u}_{k+1}^{(3)}=\max \left\{\boldsymbol{\nu}_{k}^{(3)}, 0\right\}
$$

where the maximum is to be understood in a component-wise sense; this projection has of course $O(n)$ cost.

From the observations in the previous paragraphs, the computational costs of the lines of PIDAL-TV are the following. Lines $3,4,5,9,10,11,12,13,15$, and 16 have $O(n)$ cost. Lines 6,7 , 8 , and 14 have $O(n \log n)$ cost. Thus, the computational cost of PIDAL-TV scales as $O(n \log n)$.

\section{Convergence of PIDAL-TV: Exact TV}

Convergence of PIDAL-TV is addressed by the following corollary of Theorem 1 , for the (ideal) case where $\Psi_{(\tau / \mu) \mathrm{TV}}$ (line 11) is computed exactly. The minimizations in lines 9 and 13 have the closed-form solutions given in (31) and (32).

Corollary 1: If the minimizations in lines 9, 11, and 13 of PIDAL-TV are solved exactly, then the algorithm converges to a minimizer of (10), provided one exists.

Proof: PIDAL-TV is an instance of ADMM in Fig. 1, where $f_{1}=0$ and $f_{2}$ has the form (19), with $J=3$ and the $g_{i}$ functions given in (25), which are all closed, proper, and convex. Function $f_{2}$ is, thus, also closed, proper, and convex. Matrix $\mathbf{G}=\left[\begin{array}{lll}\mathbf{K}^{T} & \mathbf{I} & \mathbf{I}\end{array}\right]^{T}$ has full column rank. The minimization in line 4 of ADMM corresponds to lines 9, 11, and 13 of PIDAL-TV; if these minimizations are solved exactly, then according to Theorem 1 , convergence to a minimizer of the objective function, if one exists, is guaranteed.

\section{Convergence of PIDAL-TV: Approximate TV}

As is well known, the (isotropic) TV denoising problem has no closed form solution, with many algorithms having been proposed to solve it (see [9], [11], [17], [44], and references therein). Here, we adopt Chambolle's algorithm [9].

Of course, in practice, Chambolle's (or any other iterative) algorithm can only run for a finite number of iterations, thus, the minimization in line 11 of PIDAL-TV can only be solved approximately. However, as stated in Theorem 1, this will not compromise the convergence of ADMM/PIDAL-TV, if the corresponding error sequence is summable. To achieve this goal, we adopt a simple procedure in which the internal variables of Chambolle's algorithm (the discrete gradient, see [9]) are initialized, in each iteration of PIDAL-TV, with those obtained in the previous iteration. We will now formalize this idea and provide experimental evidence that this procedure does produce a summable error sequence.

Let us define $\beta=\tau / \mu$ and let $(\mathbf{s}, \mathbf{q})=\widetilde{\mathbf{\Psi}}_{\beta \mathrm{TV}}^{(t)}(\mathbf{r}, \mathbf{p})$ be the result of running $t$ iterations of Chambolle's algorithm with its internal variables initialized at $\mathbf{p}$, where $\mathbf{s}$ is the obtained (denoised) image (which is approximately $\Psi_{\beta \mathrm{TV}}(\mathbf{r})$ ) and $\mathbf{q}$ the final values of the internal variables. Consider now two possible implementations of line 11 of PIDAL-TV

$$
\begin{aligned}
\text { 11(a). } \quad\left(\mathbf{u}_{k+1}^{(2, a)}, \mathbf{p}_{k+1}\right) & =\widetilde{\boldsymbol{\Psi}}_{\beta \mathrm{TV}}^{(t)}\left(\boldsymbol{\nu}_{k}^{(2)}, \mathbf{p}_{k}\right) \\
11(\mathrm{~b}) . \quad\left(\mathbf{u}_{k+1}^{(2, b)}, \cdot\right) & =\widetilde{\boldsymbol{\Psi}}_{\beta \mathrm{TV}}^{(t)}\left(\boldsymbol{\nu}_{k}^{(2)}, \mathbf{p}\right) .
\end{aligned}
$$




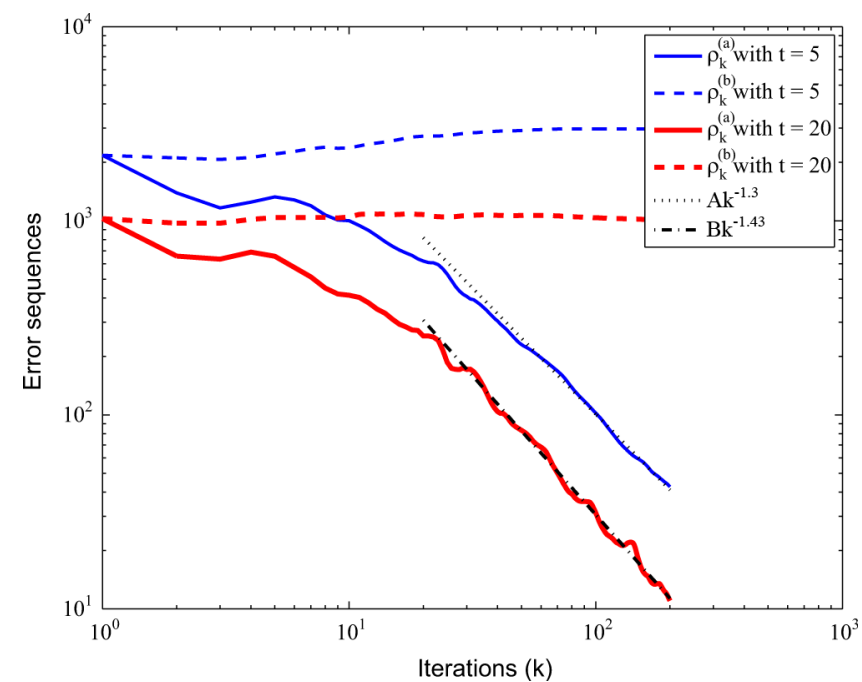

Fig. 3. Error sequences $\rho_{k}^{(a)}$ and $\rho_{k}^{(b)}$ for $t=5$ and $t=20$ (number of iterations of Chambolle's algorithm) and fitted functions of the form $A t^{\omega}$ to the sequences $\left\{\rho_{k}^{(a)}, k=20,21, \ldots 200\right\}$.

Implementation 11(a) uses the proposed internal variables initialization, whereas in 11(b) the internal variables are always initialized to the same values (usually zeros). Consider now the corresponding error sequences

$$
\begin{aligned}
& \rho_{k}^{(a)}=\left\|\mathbf{u}_{k+1}^{(2, a)}-\boldsymbol{\Psi}_{\beta \mathrm{TV}}\left(\boldsymbol{\nu}_{k}^{(2)}\right)\right\| \\
& \rho_{k}^{(b)}=\left\|\mathbf{u}_{k+1}^{(2, b)}-\boldsymbol{\Psi}_{\beta \operatorname{TV}}\left(\boldsymbol{\nu}_{k}^{(2)}\right)\right\| .
\end{aligned}
$$

Notice that since the two other minimizations (lines 9 and 13) are solved exactly, the sequences $\rho_{k}^{(a)}$ and $\rho_{k}^{(b)}$ correspond to the sequence $\rho_{k}$ in Theorem 1 .

The following experiment provides evidence that $\rho_{k}^{(a)}$ is summable, but $\rho_{k}^{(b)}$ is not. Consider the same setup as in the first experiment in [50]: the original image is a portion of the Cameraman image scaled to a maximum value of 3000 and then blurred with a Gaussian kernel of unit variance; the observed image is generated according to (1). As in [50], we set $\tau=$ 0.008 and $\mu=\tau / 50$. The number of iterations of Chambolle's algorithm is set to five or 20. To compute $\boldsymbol{\Psi}_{\beta} \mathrm{TV}\left(\boldsymbol{\nu}_{k}^{(2)}\right)$ (almost) exactly, we run 4000 iterations of Chambolle's algorithm. In Fig. 3, it is clear that the $\rho_{k}^{(b)}$ sequences are not even decreasing, let alone summable. In contrast, the sequences $\rho_{k}^{(a)}$ approach zero, for both choices of $t$. Evidence for the summability of the $\rho_{k}^{(a)}$ sequences is provided by the fact that by fitting a function of the form $A(1 / k)^{\omega}$ to the tails of these sequences (i.e., for $k=20, \ldots, 200$ ), we obtain values of $\omega$ that are larger than one ( $\omega \simeq 1.3$, for $t=5$, and $\omega \simeq 1.43$, for $t=20$ ).

In conclusion, the experiment reported in the previous paragraph, though of course not a formal proof, strongly suggests that by implementing line 11 of PIDAL-TV as in (33), the corresponding error sequence (with respect to the exact minimizations) is summable, thus, we can invoke Theorem 1 to state that PIDAL-TV converges. Moreover, this experiment shows that this is achieved with a quite small number of iterations in each call of Chambolle's algorithm. In all our experiments with PIDAL-TV, we, thus, use (33) with $t=5$.

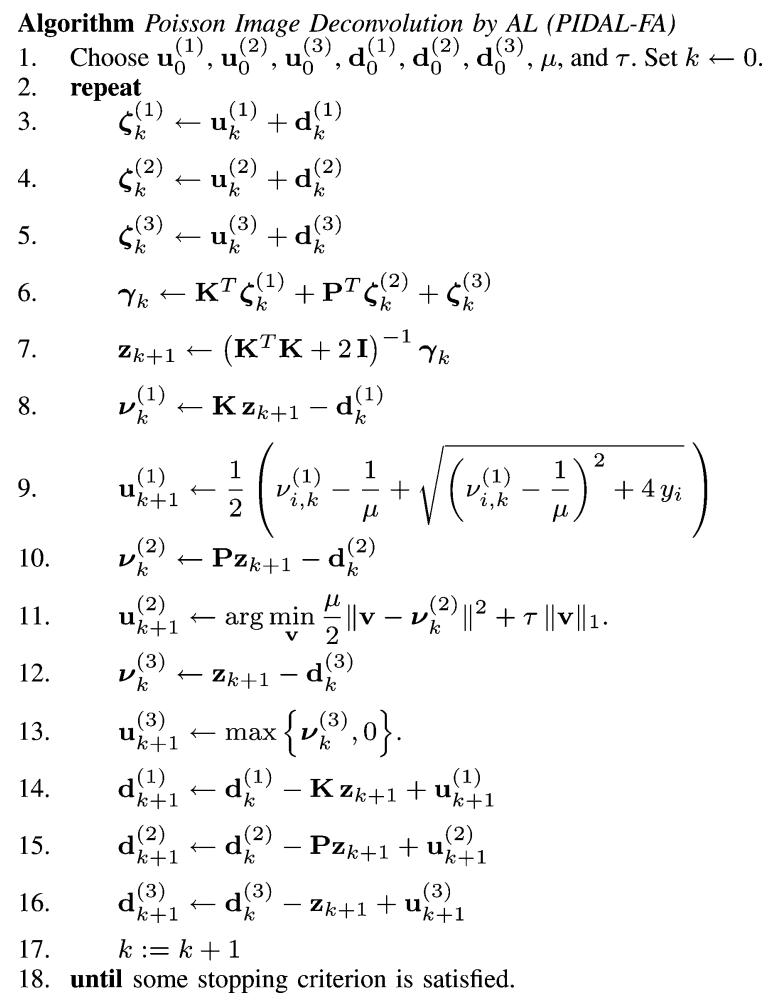

Fig. 4. PIDAL-FA algorithm.

\section{Poissonian Image Reconstruction With FRAME-BASED REGULARIZATION}

We now consider the frame-based analysis criterion (11), and the frame-based synthesis criterion (13).

\section{A. Analysis Criterion}

In this case, the objective function is given by (12), which has the form (17) with $J=3$

$$
g_{1} \equiv \mathcal{L}, \quad g_{2} \equiv \tau\|\cdot\|_{1}, \quad g_{3} \equiv \iota_{\mathbb{R}_{+}^{n}}
$$

and

$$
\mathbf{H}^{(1)} \equiv \mathbf{K}, \quad \mathbf{H}^{(2)} \equiv \mathbf{P}, \quad \mathbf{H}^{(3)} \equiv \mathbf{I} .
$$

The resulting instance of ADMM, which we call PIDAL-FA (where FA stands for "frame analysis"), is shown in Fig. 4. The matrix being inverted in line 7 results from assuming that $\mathbf{P}$ is the analysis operator of a 1-tight (Parseval) frame, thus, $\left(\mathbf{H}^{(2)}\right)^{T} \mathbf{H}^{(2)}=\mathbf{P}^{T} \mathbf{P}=\mathbf{I}$. Notice that line 7 of PIDAL-FA corresponds to (20) for the particular form of matrix $\mathbf{G}$ in this case, $\mathbf{G}=\left[\begin{array}{lll}\mathbf{K}^{T} & \mathbf{P}^{T} & \mathbf{I}\end{array}\right]^{T}$, which of course has full column rank. As in PIDAL-TV, if $\mathbf{K}$ models a convolution, the inverse $\left(\mathbf{K}^{T} \mathbf{K}+2 \mathbf{I}\right)^{-1}$ can be computed with $O(n \log n)$ cost, using the FFT [see (29)].

For most tight frames used in image processing, products by $\mathbf{P}$ and $\mathbf{P}^{T}$ correspond to the inverse and direct transforms for which fast algorithms exist. For example, in the case of translation-invariant wavelet transforms, these products can be computed using the undecimated wavelet transform with $O(n \log n)$ cost [33], [36]. Curvelets also constitute a Parseval frame for 
which fast $O(n \log n)$ implementations of the forward and inverse transform exist [7]. Yet another example of a redundant Parseval frame is provided by complex wavelets, with the corresponding direct and inverse transforms having $O(n)$ cost [32], [47]. In conclusion, for a large class of choices of $\mathbf{P}$, the cost of lines 6,10 , and 15 of PIDAL-FA is $O(n \log n)$.

The expressions in lines 9 and 13 of PIDAL-FA are similar to those in lines 9 and 13 of PIDAL-TV, respectively; see also (30)-(32).

The minimization in line 11 is, by definition, the Moreau proximity operator of the $\ell_{1}$ norm [16], which corresponds to a soft-threshold (24).

In summary, from the observations in the previous paragraphs, the computational costs of the lines of PIDAL-FA are the following. Lines 3, 4, 5, 9, 11, 12, 13, and 16 have $O(n)$ cost. Lines 6, 7, 8, 10,14, and 15 have $O(n \log n)$ cost. Thus, the computational cost of PIDAL-FA scales as $O(n \log n)$.

Finally, convergence of PIDAL-FA is addressed by the following corollary of Theorem 1.

Corollary 2: The PIDAL-FA algorithm converges to a minimizer of (11), provided one exists.

Proof: The proof is similar to, but simpler than, that of Corollary 1 , since all the minimizations involved are solved exactly in closed form. Clearly, matrix $\mathbf{G}=\left[\begin{array}{lll}\mathbf{K}^{T} & \mathbf{P}^{T} & \mathbf{I}\end{array}\right]^{T}$ has full column rank, thus, Theorem 1 guarantees convergence to a minimizer of the objective function.

\section{B. Synthesis Criterion}

In the synthesis formulation, the objective function is given by (13), which has the form (17) with $J=3$

$$
\begin{gathered}
g_{1} \equiv \mathcal{L}, \quad g_{2} \equiv \tau\|\cdot\|_{1}, \quad g_{3} \equiv \iota_{\mathbb{R}_{+}^{n}} \\
\text { and } \\
\mathbf{H}^{(1)} \equiv \mathbf{K} \mathbf{W}, \quad \mathbf{H}^{(2)} \equiv \mathbf{I}, \quad \mathbf{H}^{(3)} \equiv \mathbf{W} .
\end{gathered}
$$

The resulting ADMM algorithm, which we call PIDAL-FS (where FS stands for "frame synthesis"), is shown in Fig. 5.

Notice that line 7 of PIDAL-FS corresponds to (20) for the particular form of matrix $\mathbf{G}$ in this problem: $\mathbf{G}=\left[\begin{array}{lll}(\mathbf{K W})^{T} & \mathbf{I} & \mathbf{W}^{T}\end{array}\right]^{T}$. This matrix has of course full column rank. However, even if $\mathbf{K}$ models a periodic convolution (thus, is block circulant), the question remains of how to efficiently compute the matrix inverse in line 7 , since $\mathbf{K W}$ is not block circulant. The next paragraph shows how to sidestep this difficulty.

Consider that matrix $\mathbf{W}$ corresponds to a 1-tight (Parseval) frame, i.e., $\mathbf{W} \mathbf{W}^{H}=\mathbf{I}$, and start by noticing that $\mathbf{W}^{T} \mathbf{K}^{T} \mathbf{K} \mathbf{W}+\mathbf{I}+\mathbf{W}^{T} \mathbf{W}=\mathbf{W}^{H}\left(\mathbf{K}^{H} \mathbf{K}+\mathbf{I}\right) \mathbf{W}+\mathbf{I}$. Applying the Sherman-Morrison-Woodbury (SMW) matrix inversion formula yields

$$
\begin{aligned}
& \left(\mathbf{W}^{H}\left(\mathbf{K}^{H} \mathbf{K}+\mathbf{I}\right) \mathbf{W}+\mathbf{I}\right)^{-1} \\
& =\mathbf{I}-\mathbf{W}^{H}\left(\mathbf{W} \mathbf{W}^{H}+\left(\mathbf{K}^{H} \mathbf{K}+\mathbf{I}\right)^{-1}\right)^{-1} \mathbf{W} \\
& =\mathbf{I}-\mathbf{W}^{H}\left(\mathbf{I}+\left(\mathbf{K}^{H} \mathbf{K}+\mathbf{I}\right)^{-1}\right)^{-1} \mathbf{W} .
\end{aligned}
$$

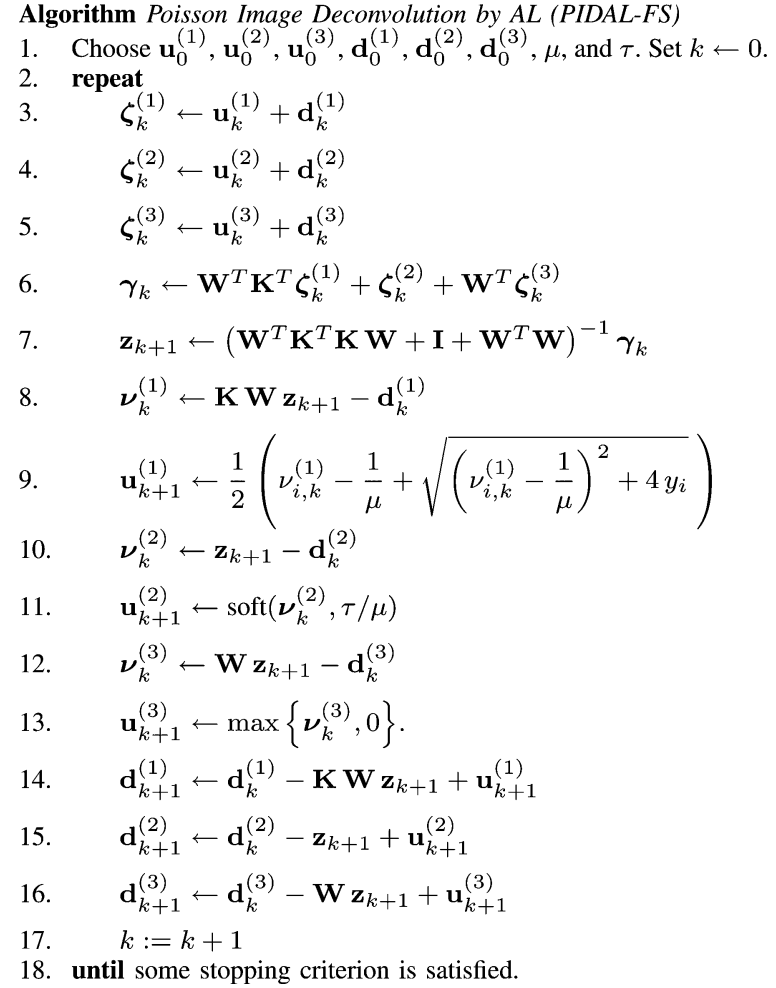

Fig. 5. PIDAL-FS algorithm.

$$
\left(\mathbf{I}+\left(\mathbf{K}^{H} \mathbf{K}+\mathbf{I}\right)^{-1}\right)^{-1}=\mathbf{U}^{H}\left(\mathbf{I}+\left(|\mathbf{D}|^{2}+\mathbf{I}\right)^{-1}\right)^{-1} \mathbf{U}
$$

where both inversions have $O(n)$ cost since $|\mathbf{D}|^{2}$ and $\mathbf{I}$ are diagonal, thus, products by the matrix in (39) have the $O(n \log n)$ cost associated to the FFT implementation of the products by $\mathbf{U}$ and $\mathbf{U}^{H}$.

The leading cost of line 7 of PIDAL-FS (given by (38)) will, thus, be either $O(n \log n)$ or the cost of the products by $\mathbf{W}^{H}$ and $\mathbf{W}$. As mentioned previously, for a large class of choices of frames, matrix-vector products by $\mathbf{W}$ and $\mathbf{W}^{H}$ have $O(n \log n)$ cost.

From the observations in the previous paragraphs, the computational costs of the lines of PIDAL-FS are the following. Lines $3,4,5,9,10,11,12,13,15$, and 16 have $O(n)$ cost. Lines 6,7 , 8 , and 14 have $O(n \log n)$ cost. Thus, the computational cost of PIDAL-FS scales as $O(n \log n)$.

Finally, convergence of PIDAL-FS is addressed by the following corollary of Theorem 1.

Corollary 3: The PIDAL-FS algorithm converges to a minimizer of (13), provided one exists.

Proof: The proof is similar to that of Corollary 2, since all the minimizations involved are solved exactly in closed form. Clearly, matrix $\mathbf{G}=\left[\mathbf{W}^{T} \mathbf{K}^{T} \mathbf{I} \mathbf{W}^{T}\right]^{T}$ has full column rank, thus, Theorem 1 guarantees convergence to a minimizer of the objective function. 
TABLE I

INITIALIZATION OF THE PIDAL ALGORITHMS

\begin{tabular}{l|c|c|c|c|c|c} 
& $\mathbf{u}_{0}^{(1)}$ & $\mathbf{u}_{0}^{(2)}$ & $\mathbf{u}_{0}^{(3)}$ & $\mathbf{d}_{0}^{(1)}$ & $\mathbf{d}_{0}^{(2)}$ & $\mathbf{d}_{0}^{(3)}$ \\
\hline PIDAL-TV & $\mathbf{y}$ & $\mathbf{y}$ & $\mathbf{y}$ & $\mathbf{0}$ & $\mathbf{0}$ & $\mathbf{0}$ \\
\hline PIDAL-FA & $\mathbf{y}$ & $\mathbf{P y}$ & $\mathbf{y}$ & $\mathbf{0}$ & $\mathbf{0}$ & $\mathbf{0}$ \\
\hline PIDAL-FS & $\mathbf{y}$ & $\mathbf{W}^{T} \mathbf{K}^{T} \mathbf{y}$ & $\mathbf{K}^{T} \mathbf{y}$ & $\mathbf{0}$ & $\mathbf{0}$ & $\mathbf{0}$ \\
\hline
\end{tabular}

\section{EXPERIMENTS}

We now report experiments where PIDAL is compared with other state-of-the-art methods, namely those proposed in [20], [27], [50]. All the algorithms are implemented in MATLAB and the experiments are carried out on a PC with a $3.0 \mathrm{GHz}$ Intel Core2Extreme $\mathrm{CPU}$, with $4 \mathrm{~Gb}$ of RAM, under Microsoft Windows Vista. Unless otherwise indicated, we manually adjust the regularization parameter $\tau$ to achieve the highest improvement in signal-noise-ratio $\left(\right.$ ISNR $\left.=10 \log _{10}\left(\|\mathbf{y}-\mathbf{x}\|_{2}^{2} /\|\widehat{\mathbf{x}}-\mathbf{x}\|_{2}^{2}\right)\right)$. The PIDAL algorithms are initialized as shown in Table I.

According to Theorem 1, ADMM (thus, PIDAL) converges for any choice of $\mu>0$. However, this parameter does influence the speed of the algorithms. To our knowledge, there is no work on methods to choose this parameter for optimal speed; in our experiments, we use the following rule of thumb, found to achieve satisfactory results: $\mu=60 \tau / M$, where $M$ is the maximum intensity of the original image. We have observed that the results do not change significantly if this parameter is changed to one order of magnitude below or above this choice.

\section{A. Comparison With [50]}

We begin by comparing with the algorithms (PIDSplit and PIDSplit+) proposed in [50], which (as acknowledged by the authors of [50]) is based upon the earlier version of PIDAL-TV [25]. The setup was already described in Section IV-D: the original image is a portion $(84 \times 84)$ of the Cameraman image, scaled to a maximum value of 3000 and blurred with a Gaussian kernel of unit variance; the observed image is generated according to (1); the regularization parameter is set to $\tau=0.008$. In the experiments reported in [50], the TV denoising step of PIDAL-TV is implemented by an inner iterative algorithm with a tight stopping criterion based upon the change between two consecutive images. Our implementation of PIDAL-TV, as explained in Section IV-D, uses a small and fixed number of iterations (just 5) of Chambolle's algorithm, which is initialized as explained in that section. Because PIDSplit and PIDSplit+ have no inner loop, each of its iterations is roughly equivalent to one iteration of PIDAL-TV with just one iteration of Chambolle's algorithm. In [50], PIDSplit and PIDSplit+ were run for 2150 iterations; we, thus, run PIDAL-TV for $2150 / 5=$ 430 iterations, corresponding to roughly the same amount of computation. Fig. 6 shows the evolution of the mean absolute error(MAE $\left.=\|\widehat{\mathbf{x}}-\mathbf{x}\|_{1} / n\right)$ and ISNR along the first 160 iterations of PIDAL-TV (as well as elapsed time); it is clear that convergence is achieved after less than 140 iterations ( $4.3 \mathrm{~s}$ in our computer). This is dramatically less than what is reported in [50] for PIDAL-TV; in terms of iterations of PIDSplit and PIDSplit+, this corresponds to approximately $150 * 5=750$ iterations, thus, also much less than the 2150 iterations (11 s) re-

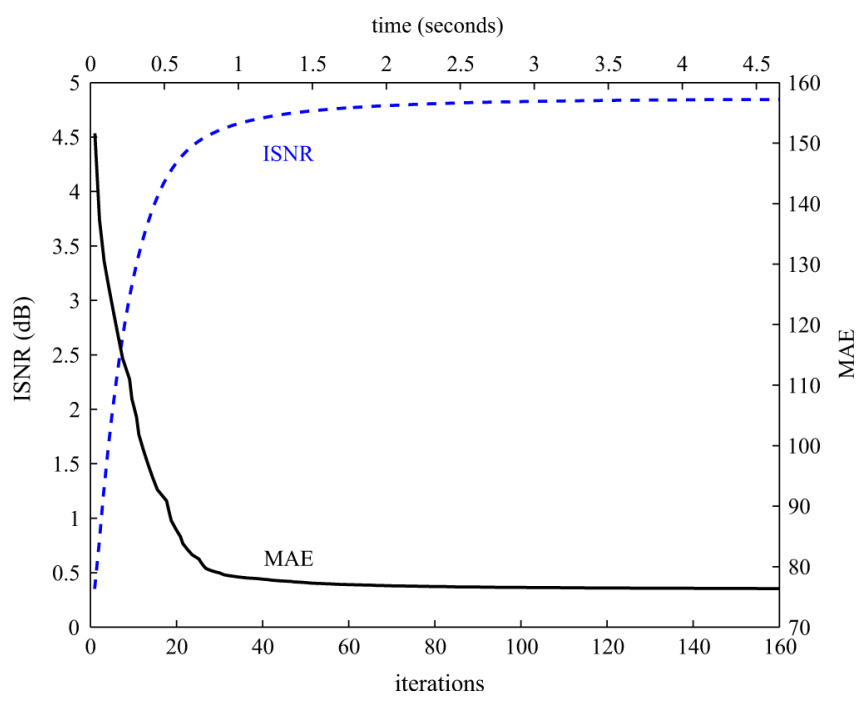

Fig. 6. Evolution of the MAE and ISNR along the iterations and elapsed time of PIDAL-TV, for the experiment of Section VI-A.

ported in that work. Finally, Fig. 7 shows the original, observed, and restored images; as expected, the image estimates produced by PIDSplit and PIDAL-TV are very similar.

Finally, we also tested PIDAL-FA and PIDAL-FS on the same example, using a fully redundant Haar frame. The plots of ISNR and MAE are presented in Figs. 8 and 9, while the estimated images are shown in Fig. 10.

These results show that, in this example, PIDAL-FA performs slightly better than PIDAL-TV in terms of ISNR and similarly in terms of MAE, with PIDAL-FA achieving its best estimate faster than PIDAL-TV. The synthesis-based criterion implemented by PIDAL-FS is a little worse in terms of both ISNR and MAE, and PIDAL-FS also takes longer than PIDAL-FA to achieve its best estimate. This poorer performance of the synthesis formulation (in line with recent results in [48]) was also found in all the experiments reported in the following, so we will only present results for PIDAL-TV and PIDAL-FA.

\section{B. Comparison With [27]}

The next experiment follows [27]: the original image is the complete $(256 \times 256)$ Cameraman, scaled to a maximum value of 17600 , the blur is $9 \times 9$ uniform. As in the experiment reported in the previous subsection, this is a high SNR situation. Fig. 11 shows the evolution of the MAE and ISNR along the execution of PIDAL-TV; it is clear that convergence is achieved after about 160 iterations ( $25 \mathrm{~s}$ in our computer). A detail of the blurred, and estimated images (from [27] and using PIDAL-TV and PIDAL-FA) are shown in Fig. 12. Although the TV and FA regularizers are considerably simpler than the locally adaptive approximation techniques used in [27], both PIDAL-TV and PIDAL-FA achieve higher ISNR values $(7.0$ and $6.95 \mathrm{~dB}$, respectively) than that reported in [27] $(6.61 \mathrm{~dB})$.

\section{Comparison With [20]}

In the last set of experiments we compare our approach with another recent state-of-the-art algorithm (herein referred 

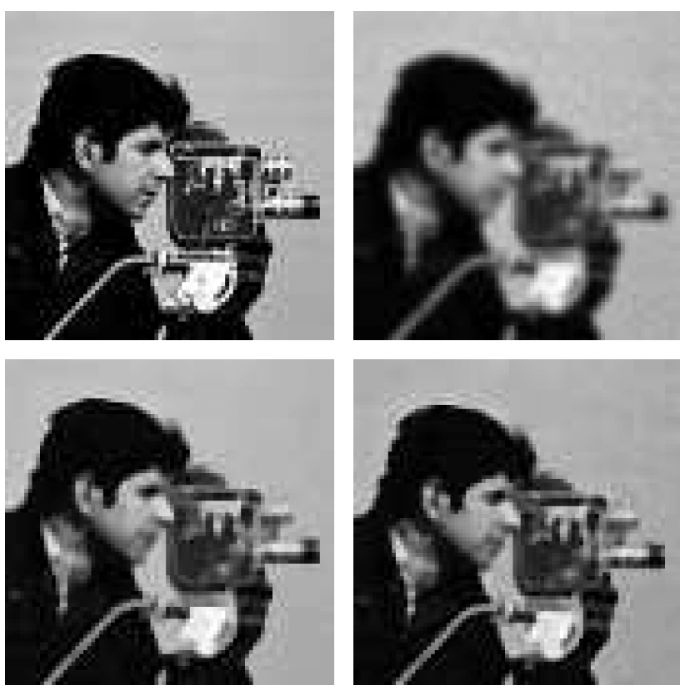

Fig. 7. Experiment of Section VI-A. Top row: (left) original; (right) blurred and noisy image. Bottom row: estimate from [50]; estimate by PIDAL-TV (ISNR = $4.8 \mathrm{~dB})$.

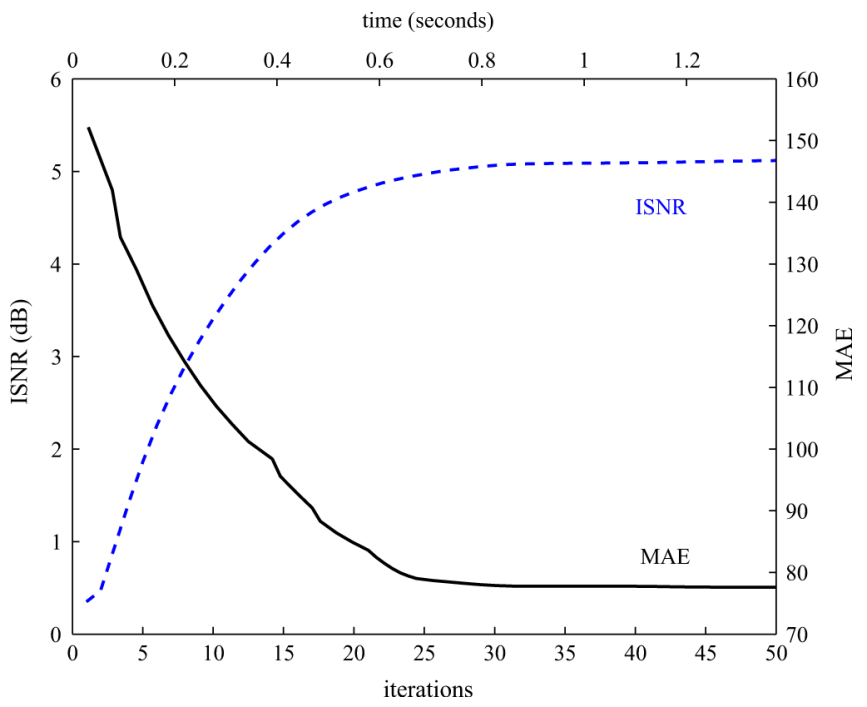

Fig. 8. Experiment of Section VI-A. Evolution of the MAE and ISNR along the iterations and elapsed time of PIDAL-FA.

to as DFS), proposed in [20], for which the MATLAB implementation is available at www.greyc.ensicaen.fr/ fdupe. That work includes comparisons with other methods, namely: Richardson-Lucy with multiresolution support wavelet regularization (RL-MRS) [52]; fast translation invariant tree-pruning reconstruction (FTITPR) [58]; Richardson-Lucy with total variation regularization (RL-TV) [18]. The results in [20] show that the algorithm therein proposed generally achieves better performance (i.e., lower MAE) than the others, except for one of the images (a microscopy cell image) where RL-MRS outperforms DFS. For this reason, we will report results comparing PIDAL-TV and PIDAL-FA versus DFS and RL-MRS. For PIDAL-FA, we use a redundant Haar frame for the Cameraman image and Daubechies-4 for the other images. As in [20], the

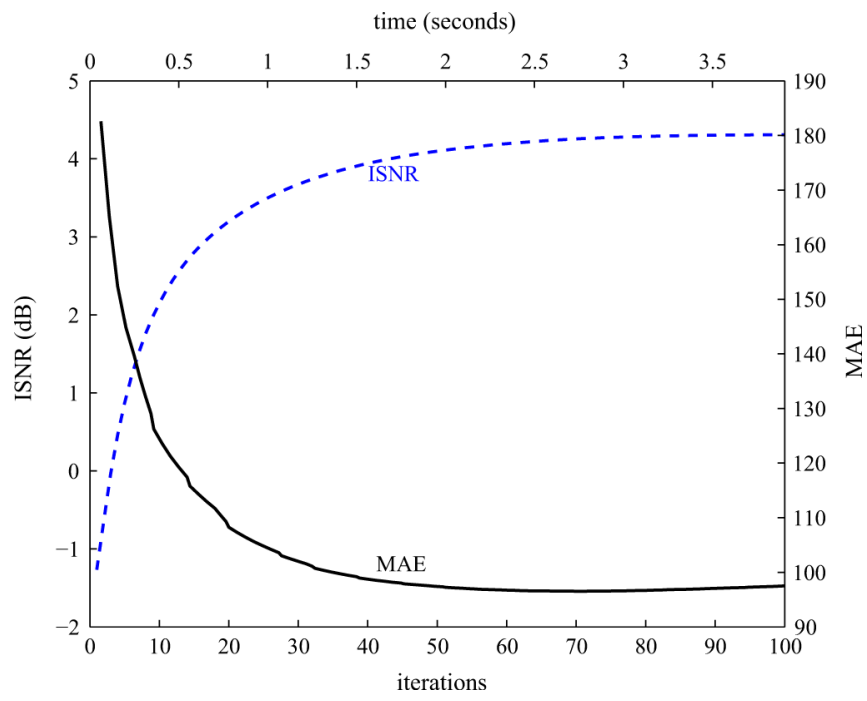

Fig. 9. Experiment of Section VI-A. Evolution of the MAE and ISNR along the iterations and elapsed time of PIDAL-FS.
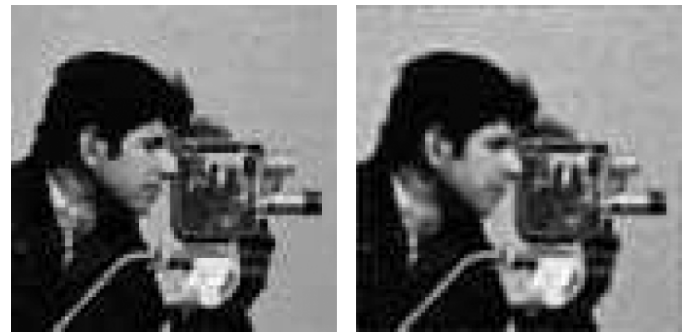

Fig. 10. Experiment of Section VI-A. Left: PIDAL-FA estimate (ISNR = $5.3 \mathrm{~dB}$ ). Right: PIDAL-FS estimate (ISNR $=4.3 \mathrm{~dB}$ ).

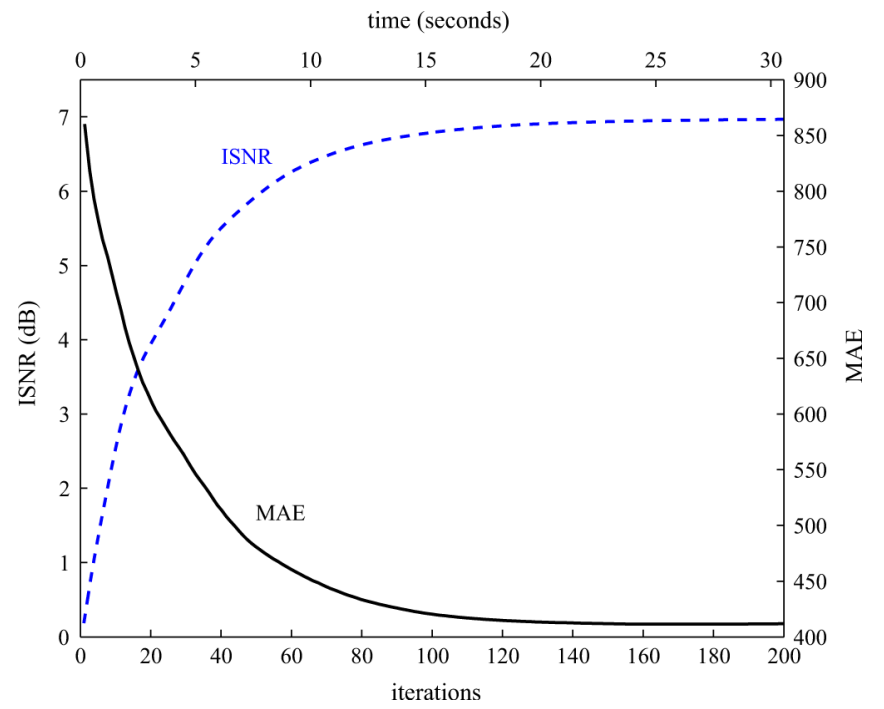

Fig. 11. Experiment of Section VI-B. Evolution of the MAE and ISNR along the iterations and elapsed time of PIDAL-TV.

original images are scaled to a maximum value $M$, belonging to $5,30,100,255$, and then blurred by a $7 \times 7$ uniform filter.

The DFS algorithm does not include a stopping criterion, with the results reported in [20] having been obtained by running a fixed number (200) of iterations. In order to compare the running times of PIDAL-TV, PIDAL-FA, and DFS, we run DFS 
TABLE II

RESUlts of the COMPARISON OF PIDAL-TV AND PIDAL-FA With the Algorithms PRoposed IN [20] AND [52] (Average Over 10 Runs); THE RePorted Times ARE IN SECONDS

\begin{tabular}{|c|c|c|c|c|c|c|c|c|c|c|c|}
\hline & & \multicolumn{3}{|c|}{ PIDAL-TV } & \multicolumn{3}{|c|}{ PIDAL-FA } & \multicolumn{3}{|c|}{ DFS [20] } & RL-MRS [52] \\
\hline Image & $M$ & MAE & iterations & time & MAE & iterations & time & MAE & iterations & time & MAE \\
\hline Cameraman & 5 & 0.27 & 120 & 22 & 0.26 & 70 & 13 & 0.35 & 6 & 4.5 & 0.37 \\
\hline Cameraman & 30 & 1.29 & 51 & 9.1 & 1.22 & 39 & 7.4 & 1.47 & 98 & 75 & 2.06 \\
\hline Cameraman & 100 & 3.99 & 33 & 6.0 & 3.63 & 36 & 6.8 & 4.31 & 426 & 318 & 5.58 \\
\hline Cameraman & 255 & 8.99 & 32 & 5.8 & 8.45 & 37 & 7.0 & 10.26 & 480 & 358 & 12.3 \\
\hline Neuron & 5 & 0.17 & 117 & 3.6 & 0.18 & 66 & 2.9 & 0.19 & 6 & 3.9 & 0.19 \\
\hline Neuron & 30 & 0.68 & 54 & 1.8 & 0.77 & 44 & 2.0 & 0.82 & 161 & 77 & 0.95 \\
\hline Neuron & 100 & 1.75 & 43 & 1.4 & 2.04 & 41 & 1.8 & 2.32 & 427 & 199 & 2.88 \\
\hline Neuron & 255 & 3.52 & 43 & 1.4 & 3.47 & 42 & 1.9 & 5.25 & 202 & 97 & 6.31 \\
\hline Cell & 5 & 0.12 & 56 & 10 & 0.11 & 36 & 7.6 & 0.12 & 6 & 4.5 & 0.12 \\
\hline Cell & 30 & 0.57 & 31 & 6.5 & 0.54 & 39 & 8.2 & 0.56 & 85 & 64 & 0.47 \\
\hline Cell & 100 & 1.71 & 85 & 15 & 1.46 & 31 & 6.4 & 1.72 & 215 & 162 & 1.37 \\
\hline Cell & 255 & 3.77 & 89 & 17 & 3.33 & 34 & 7.0 & 5.45 & 410 & 308 & 3.10 \\
\hline
\end{tabular}
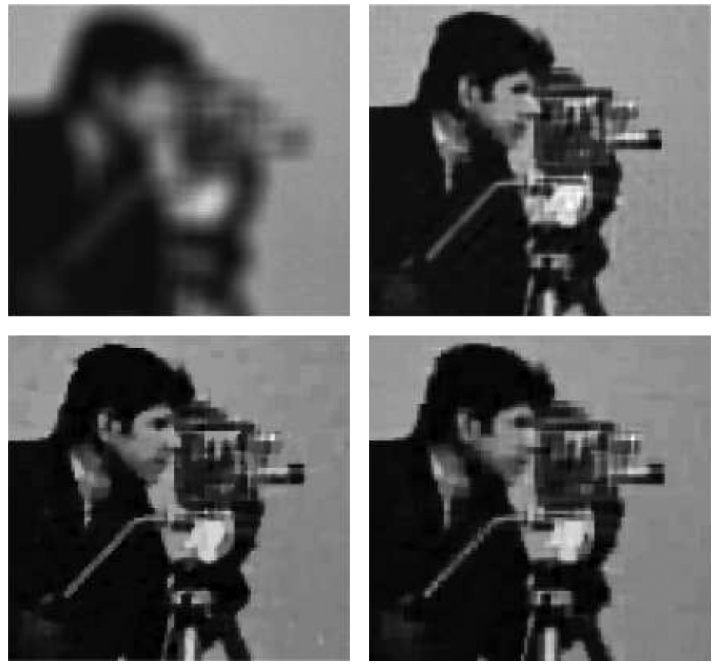

Fig. 12. Experiment of Section VI-B. Top row: blurred noisy image (left) and estimate from [27](ISNR $=6.61 \mathrm{~dB})$. Bottom row: PIDAL-TV estimate (left, ISNR $=7.0 \mathrm{~dB}$ ); PIDAL-FA estimate (right, ISNR $=6.95 \mathrm{~dB}$ ).

until the MAE decreases less than $0.01 \%$ between two consecutive iterations. Our algorithms are stopped when the following condition is met:

$$
\frac{\left\|\mathbf{z}_{k}-\mathbf{z}_{k-1}\right\|_{2}}{\left\|\mathbf{z}_{k-1}\right\|_{2}} \leq \delta
$$

with $\delta=0.005$ if $M=5$ and $\delta=0.001$ in all the other cases. Notice that this favors DFS, since a stopping criterion based upon MAE is not applicable in practice due to the absence of the original image.

The results reported in Table II show that, in nine out of the 12 experiments, either PIDAL-TV or PIDAL-FA achieves the lowest MAE. Notice however, that the main goal of this paper was not to introduce a new restoration criterion aiming at obtaining the lowest possible MAE (or any other performance measure), but rather to introduce algorithms to solve the optimization problems resulting from variational formulations of Poissonian image restoration. In terms of computational efficiency, PIDAL-TV and PIDAL-FA are clearly faster than the DRS algorithm, except in the very low SNR situations $(M=5)$ for two of the images (Cameraman and Cell).

\section{CONCLUDING REMARKS}

We have propose new algorithms to handle the optimization problems resulting from regularization approaches to the restoration of Poissonian images. These optimization problems include several difficulties: the Poisson log-likelihood is nonquadratic and its gradient is not Lipschitz; the state-of-the-art regularizers are nonsmooth; there is a nonnegativity constraint. We have started by presenting sufficient conditions for existence and uniqueness of solutions of these optimization problems, for the following regularizers: total-variation, frame-based analysis, and frame-based synthesis. These problems were handled by adapting the alternating direction method of multipliers (ADMM) to their particular forms. This adaptation is based upon a new way of using ADMM to deal with problems in which the objective function is a linear combination of convex terms, which can be used in many other problems. We gave sufficient conditions for convergence and proved that these are met in the considered cases. Finally, we have experimentally compared the proposed algorithms against competing techniques, showing that they achieve state-of-the-art performance both in terms of speed and restoration accuracy.

\section{APPENDIX A \\ CONVEX ANALYSIS}

We very briefly review some basic convex analysis results used in this paper. For more details see [16], [61].

Consider a function $f: \mathcal{X} \rightarrow \overline{\mathbb{R}}=\mathbb{R} \cup\{-\infty,+\infty\}$, where $\overline{\mathbb{R}}$ is called the extended real line, and $\mathcal{X}$ is a real Hilbert space. The domain of function $f$ is the set $\operatorname{dom}(f)=\{\mathbf{x}: f(\mathbf{x})<+\infty\}$.

The function $f$ is convex if $f(\alpha \mathbf{u}+(1-\alpha) \mathbf{v}) \leq \alpha f(\mathbf{u})+$ $(1-\alpha) f(\mathbf{v})$, for any $\mathbf{u}, \mathbf{v} \in \mathcal{X}$ and any $\alpha \in[0,1]$. Convexity is said to be strict if the inequality holds strictly $(<)$ for any $\mathbf{u}, \mathbf{v} \in \operatorname{dom}(f)$ and $\alpha \in] 0,1[$.

The function is called proper if it is not equal to $+\infty$ everywhere and is never equal to $-\infty$.

The function $f$ is lower semicontinuous (lsc) at $\mathbf{v}$ if

$$
\lim _{\delta \searrow 0} \inf _{\mathbf{x} \in B(\mathbf{v}, \delta)} f(\mathbf{x}) \geq f(\mathbf{v})
$$

where $B(\mathbf{v}, \delta)=\{\mathbf{x}:\|\mathbf{x}-\mathbf{v}\| \leq \delta\}$ is the $\delta$-ball around $\mathbf{v}$, and $\|\cdot\|$ is the norm in the Hilbert space $\mathcal{X}$. A function is called lsc if it is lsc at every point of its domain. 
A function $f$ is called coercive if it verifies $\lim _{\|\mathbf{x}\| \rightarrow \infty} f(\mathbf{x})=+\infty$. Proper, lsc, coercive functions play a key role in optimization via the following theorem [16]:

Theorem 2: If $f$ is a proper, lsc, coercive, convex function, then the set $\arg \min _{\mathbf{x} \in \mathcal{X}} f(\mathbf{x})$ is nonempty.

The next theorem concerns strictly convex functions.

Theorem 3: If $f$ is a strictly convex function, the set $\arg \min _{\mathbf{x} \in \mathcal{X}} f(\mathbf{x})$ possesses at most one element.

\section{APPENDIX B \\ MOREAU PROXIMITY OPERATORS}

Consider a function $\Phi: \mathcal{X} \rightarrow \overline{\mathbb{R}}$, where $\mathcal{X}$ is a real Hilbert space (with norm denoted by $\|\cdot\|$ ), assumed to be convex, lsc, and proper (see Appendix A for definitions and implications of these properties). Consider also the function $d_{\mathbf{y}}: \mathcal{X} \rightarrow \mathbb{R}$, defined as

$$
d_{\mathbf{y}}(\mathbf{x})=\|\mathbf{x}-\mathbf{y}\|^{2} .
$$

Clearly, for any $\mathbf{y}$, the function $d_{\mathbf{y}}$ is convex, lsc, proper, and coercive, so, thus, is the function $f:=d_{\mathbf{y}}+\Phi$. According to Theorem 2 (Appendix A), the set of minimizers of $f$ is not empty. Moreover, since $d_{\mathbf{y}}$ is strictly convex, $f$ is strictly convex, thus, the minimizer of $f$ is unique (see Theorem 3 in Appendix A). From these facts, the so-called Moreau proximity operator (see [12], [14]-[16], [37], and [38]) $\Psi_{\Phi}: \mathcal{X} \rightarrow \mathcal{X}$

$$
\Psi_{\Phi}(\mathbf{y})=\arg \min _{\mathbf{x}}\left\{\frac{d_{\mathbf{y}}^{2}(\mathbf{x})}{2}+\Phi(\mathbf{x})\right\}
$$

is well defined. Many examples of Moreau proximity operators can be found in [12] and [14].

\section{REFERENCES}

[1] F. Abramovich and B. Silverman, "Wavelet decomposition approaches to statistical inverse problems," Biometrika, vol. 85, pp. 115-129, 1998.

[2] A. Antoniadis and J. Bigot, "Poisson inverse problems," Ann. Statist., vol. 34, pp. 1811-1825, 2006.

[3] G. Aubert and J. Aujol, "A variational approach to remove multiplicative noise," SIAM J. Appl. Math., vol. 68, no. 4, pp. 925-946, 2008.

[4] A. Beck and M. Teboulle, "A fast iterative shrinkage-thresholding algorithm for linear inverse problems," SIAM J. Imag. Sci., vol. 2, pp. 183-202, 2009.

[5] J. Bioucas-Dias and M. Figueiredo, "A new TwIST: Two-step iterative shrinkage/thresholding algorithms for image restoration," IEEE Trans. Image Process., vol. 16, no. 12, pp. 2992-3004, Dec. 2007.

[6] J. Bioucas-Dias and M. Figueiredo, "Multiplicative noise removal using variable splitting and constrained optimization," IEEE Trans. Image Process., vol. 19, no. 7, pp. 1720-1730, Jul. 2010.

[7] E. Candès, L. Demanet, D. Donoho, and L. Ying, "Fast discrete curvelet transforms," SIAM J. Multiscale Model. Sim., vol. 5, pp. 861-899, 2005.

[8] L. Cavalier and J.-Y. Koo, "Poisson intensity estimation for tomographic data using a wavelet shrinkage approach," IEEE Trans. Inf. Theory, vol. 48, no. 10, pp. 2794-2802, Oct. 2002.

[9] A. Chambolle, "An algorithm for total variation minimization and applications," J. Math. Imag. Vis., vol. 20, pp. 89-97, 2004.
[10] A. Chambolle and P.-L. Lions, "Image recovery via total variation minimization and related problems," Numer. Math., vol. 76, pp. 167-188, 1997.

[11] T. Chan, S. Esedoglu, F. Park, and A. Yip, "Recent developments in total variation image restoration," in Mathematical Models of Computer Vision. New York: Springer-Verlag, 2005.

[12] C. Chaux, P. Combettes, J.-C. Pesquet, and V. Wajs, "A variational formulation for frame-based inverse problems," Inv. Probl., vol. 23, pp. 1495-1518, 2007.

[13] T. Chan, G. Golub, and P. Mulet, "A nonlinear primal-dual method for total variation-based image restoration," SIAM J. Sci. Comput., vol. 20, pp. 1964-1977, 1999.

[14] P. Combettes and J.-C. Pesquet, "Proximal thresholding algorithm for minimization over orthonormal bases," SIAM J. Optim., vol. 18, pp. 1351-1376, 2007.

[15] P. Combettes and J.-C. Pesquet, "A Douglas-Rachford splitting approach to nonsmooth convex variational signal recovery," IEEE J. Sel. Topics Signal Process., vol. 1, no. 4, pp. 564-574, Dec. 2007.

[16] P. Combettes and V. Wajs, "Signal recovery by proximal forward-backward splitting," SIAM J. Multiscale Model. Sim., vol. 4, pp. 1168-1200, 2005.

[17] J. Dahl, P. Hansen, S. Jensen, and T. Jensen, "Algorithms and software for total variation image reconstruction via first-order methods," Numer. Algorithms, vol. 53, pp. 67-92, 2010.

[18] N. Dey, L. Blanc-Feraud, C. Zimmer, P. Roux, Z. Kam, J. Olivo-Marin, and J. Zerubia, "Richardson-Lucy algorithm with TV regularization for 3D confocal microscope deconvolution," Microsc. Res. Tech., vol. 69, pp. 260-266, 2006.

[19] D. Donoho, "Nonlinear solution of linear inverse problems by waveletvaguelette decomposition," Appl. Comput. Harmon. Anal., vol. 2, pp. 101-126, 1995.

[20] F.-X. Dupé, J. Fadili, and J.-L. Starck, "A proximal iteration for deconvolving Poisson images using sparse representations," IEEE Trans. Image Process., vol. 18, no. 2, pp. 310-321, Feb. 2009.

[21] J. Eckstein and D. Bertsekas, "On the Douglas-Rachford splitting method and the proximal point algorithm for maximal monotone operators," Math. Program., vol. 5, pp. 293-318, 1992.

[22] M. Elad, P. Milanfar, and R. Rubinstein, "Analysis versus synthesis in signal priors," Inv. Probl., vol. 23, pp. 947-968, 2007.

[23] E. Esser, "Applications of Lagrangian-based alternating direction methods and connections to split Bregman computational and applied math,” Univ. California, Los Angeles, 2009, Tech. Rep. 09-31.

[24] J. Fessler and A. Hero , III, "Penalized maximum-likelihood image reconstruction using space-alternating generalized EM algorithms," IEEE Trans. Image Process., vol. 4, no. 10, pp. 1417-29, Oct. 1995.

[25] M. Figueiredo and J. Bioucas-Dias, "Deconvolution of Poissonian images using variable splitting and augmented Lagrangian optimization," in Proc. IEEE Workshop Statist. Signal Process., Cardiff, U.K., 2009, pp. 733-736.

[26] M. Figueiredo and R. Nowak, "An EM algorithm for wavelet-based image restoration," IEEE Trans. Image Process., vol. 12, no. 8, pp. 906-916, Aug. 2003

[27] A. Foi, S. Alenius, M. Trimeche, V. Katkovnik, and K. Egiazarian, "A spatially adaptive Poissonian image deblurring," in Proc. IEEE Int. Conf. Image Process., Genova, Italy, 2005, vol. 1, pp. 925-928.

[28] D. Gabay and B. Mercier, "A dual algorithm for the solution of nonlinear variational problems via finite-element approximations," Comput. Math. Appl., vol. 2, pp. 17-40, 1976.

[29] R. Glowinski and A. Marroco, "Sur l'approximation, par elements finis d'ordre un, et la resolution, par penalisation-dualité d'une classe de problemes de dirichlet non lineares," Rev. Française d'Automatique, Inf. Recherche Opérationelle, vol. 9, pp. 41-76, 1975.

[30] T. Goldstein and S. Osher, "The split Bregman method for L1 regularized problems, computational and applied math," Univ. California, Los Angeles, 2008, Tech. Rep. 08-29.

[31] M. Hestenes, "Multiplier and gradient methods," J. Optim. Theory Appl., vol. 4, pp. 303-320, 1969.

[32] N. Kingsbury, "Complex wavelets for shift invariant analysis and filtering of signals," J. Appl. Comput. Harmon. Anal., vol. 10, pp. 234-253, 2001.

[33] M. Lang, H. Guo, J. Odegard, C. Burrus, and R. Wells, "Noise reduction using an undecimated discrete wavelet transform," IEEE Signal Process. Lett., vol. 3, no. 1, pp. 10-12, Jan. 1996. 
[34] T. Le, R. Chartrand, and T. Asaki, "A variational approach to reconstructing images corrupted by Poisson noise," J. Math. Imag. Vis., vol. 27, pp. 257-263, 2007.

[35] M. Lustig, D. Donoho, and J. Pauly, "Sparse MRI: The application of compressed sensing for rapid MR imaging," Magnetic Resonance Med., vol. 58, pp. 1182-1195, 2007.

[36] S. Mallat, A Wavelet Tour of Signal Processing. New York: Academic, 2009.

[37] J.-J. Moreau, "Propriétés des applications prox," Comptes Rendus de l'Académie des Sciences (Série A, Mathématiques), vol. 256, pp. 1069-1071, 1963.

[38] J.-J. Moreau, "Proximité et dualité dans un espace hilbertien," Bull. Soc. Math. France, vol. 93, pp. 273-299, 1965.

[39] J. Nocedal and S. Wright, Numerical Optimization. New York: Springer-Verlag, 2006.

[40] R. Nowak, "Optimal signal estimation using cross-validation," IEEE Signal Process. Lett., vol. 4, no. 1, pp. 23-25, Jan. 1997.

[41] R. Nowak and R. Baraniuk, "Wavelet-domain filtering for photon imaging systems," IEEE Trans. Image Process., vol. 8, no. 5, pp. 666-678, May 1999.

[42] R. Nowak and E. Kolaczyk, "A Bayesian multiscale framework for Poisson inverse problems," IEEE Trans. Inf. Theory, vol. 46, pp. $1811-1825,2000$

[43] R. Nowak and M. Thul, "Wavelet-vaguelette restoration in photon-limited imaging," in Proc. IEEE Int. Conf. Acoust., Speech, Signal Process., 1998, vol. 5, pp. 2869-2872.

[44] S. Osher, L. Rudin, and E. Fatemi, "Nonlinear total variation based noise removal algorithms," Phys. D, vol. 60, pp. 259-268, 1992.

[45] M. Powell, "A method for nonlinear constraints in minimization problems," in Optimization, R. Fletcher, Ed. New York: Academic, 1969, pp. 283-298.

[46] P. Sarder and A. Nehorai, "Deconvolution method for 3-D fluorescence microscopy images," IEEE Signal Process. Mag., vol. 23, no. 3, pp. 32-45, May 2006.

[47] I. Selesnick, "Hilbert transform pairs of wavelet bases," IEEE Signal Process. Lett., vol. 8, no. 6, pp. 170-173, Jun. 2001.

[48] I. Selesnick and M. Figueiredo, "Signal restoration with overcomplete wavelet transforms: comparison of analysis and synthesis priors," Proc. SPIE, vol. 7446, pp. 0D-15D, 2009.

[49] S. Setzer, "Split Bregman algorithm, Douglas-Rachford splitting, and frame shrinkage," in Proc. 2nd Int. Conf. Scale Space Variat. Meth. Comput. Vis., 2009, vol. 5567, pp. 464-476.

[50] S. Setzer, G. Steidl, and T. Teuber, "Deblurring Poissonian images by split Bregman techniques," J. Vis. Commun. Image Represent., 2010.

[51] J.-L. Starck and F. Murtagh, Astronomical Image and Data Analysis. New York: Springer-Verlag, 2006.

[52] J.-L. Starck, A. Bijaoui, and F. Murtagh, "Multiresolution support applied to image filtering and deconvolution," Comput. Vis. Graph. Image Process., vol. 57, pp. 420-431, 1995.

[53] J.-L. Starck, M. Nguyen, and F. Murtagh, "Wavelets and curvelets for image deconvolution: A combined approach," Signal Process., vol. 83, pp. 2279-2283, 2003.

[54] G. Steidl and T. Teuber, "Removing multiplicative noise by DouglasRachford splitting methods," J. Math. Imag. Vis., vol. 36, pp. 168-184, 2010.

[55] K. Timmermann and R. Nowak, "Multiscale modeling and estimation of Poisson processes with application to photon-limited imaging," IEEE Trans. Inf. Theory, vol. 45, no. 3, pp. 846-862, Apr. 1999.

[56] Y. Wang, J. Yang, W. Yin, and Y. Zhang, "A new alternating minimization algorithm for total variation image reconstruction," SIAM J. Imag. Sci., vol. 1, pp. 248-272, 2008.

[57] R. Willett and R. Nowak, "Platelets: A multiscale approach for recovering edges and surfaces in photon-limited medical imaging," IEEE Trans. Med. Imag., vol. 22, no. 3, pp. 332-350, Mar. 2003.
[58] R. Willett and R. Nowak, "Fast multiresolution photon-limited image reconstruction," in IEEE Int. Symp. Biomed. Imag., 2004, vol. 2, pp. 1192-1195.

[59] S. Wright, R. Nowak, and M. Figueiredo, "Sparse reconstruction by separable approximation,” IEEE Trans. Signal Process., vol. 57, no. 7, pp. 2479-2493, Jul. 2009.

[60] W. Yin, S. Osher, D. Goldfarb, and J. Darbon, "Bregman iterative algorithms for $\ell_{1}$-minimization with applications to compressed sensing," SIAM J. Imag. Sci., vol. 1, pp. 143-168, 2008.

[61] C. Zalinescu, Convex Analysis in General Vector Spaces. Singapore: World Scientific, 2002.

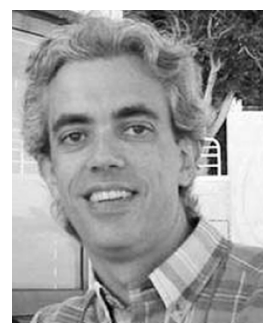

Mário A. T. Figueiredo (S'87-M'95-SM'00-F'10) received the E.E., M.Sc., Ph.D., and "Agregado" degrees in electrical and computer engineering, all from Instituto Superior Técnico (IST), the engineering school of the Technical University of Lisbon (TULisbon), Portugal, in 1985, 1990, 1994, and 2004, respectively.

Since 1994, he has been with the faculty of the Department of Electrical and Computer Engineering, IST. He is also area coordinator at Instituto de Telecomunicações, a private not-for-profit research institution. He has spent sabbatical leaves at the Department of Computer Science and Engineering, Michigan State University, and the Department of Electrical and Computer Engineering, University of Wisconsin-Madison, in 1998 and 2005, respectively. His research interests include image processing and analysis, pattern recognition, statistical learning, and optimization.

Dr. Figueiredo is a Fellow of the IAPR (International Association for Pattern Recognition) and a member of the Image, Video, and Multidimensional Signal Processing Technical Committee of the IEEE. He received the 1995 Portuguese IBM Scientific Prize and the 2008 UTL/Santander-Totta Scientific Prize $\mathrm{He}$ is/was associate editor of the following journals: IEEE TRANSACTIONS ON IMAge PROCESSING, IEEE TRANSACTIONS ON PATTERN ANALYSIS AND Machine InTELligence, IEEE TRANSACtions on Mobile Computing, Pattern Recognition, and Signal Processing. He was a co-chair of the 2001 and 2003 Workshops on Energy Minimization Methods in Computer Vision and Pattern Recognition, and program/technical committee member of many international conferences.

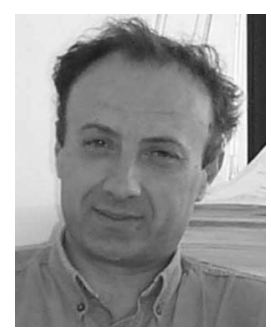

José M. Bioucas-Dias (S'87-M'95) received the E.E., M.Sc., Ph.D., and "Agregado" degrees, all in electrical and computer engineering, from Instituto Superior Técnico (IST), the Engineering School of the Technical University of Lisbon (TULIsbon), Portugal, in 1985, 1991, 1995, and 2007, respectively.

Since 1995, he has been with the Department of Electrical and Computer Engineering, IST. He is also a Senior Researcher with the Communication Theory and Pattern Recognition Group of the Institute of Telecommunications, a private not-for-profit research institution. His research interests include signal and image processing, pattern recognition, optimization, and remote sensing.

Dr. Bioucas-Dias is involved in several national and international research projects and networks, including the Marie Curie Actions "Hyperspectral Imaging Network (HYPER-I-NET)" and the "European Doctoral Program in Signal Processing (SIGNAL)." He is an Associate Editor of IEEE TRANSACTIONS ON IMAGE PROCESSING, was an Associate Editor of the IEEE TRANSACTIONS ON CIRCUITS AND SYSTEMS, and a guest editor of a special issue of the IEEE TRAnsactions on Geoscience and REMOte SENSING. $\mathrm{He}$ has been a member of program/technical committees of several international conferences, including CVPR, ICPR, ICIAR, IGARSS, ICIP, SPIE, EMMCVPR, ISVC, and WHISPERS 